Article

\title{
Effects of Oral Glutamine Supplementation on Early Postnatal Muscle Morphology in Low and Normal Birth Weight Piglets
}

\author{
Yaolu Zhao ${ }^{1}$, Elke Albrecht ${ }^{1, * \mathbb{C}}$, Quentin L. Sciascia ${ }^{2}{ }^{\mathbb{D}}$, Zeyang Li ${ }^{2}$, Solvig Görs ${ }^{2}$, \\ Johannes Schregel $^{2}$, Cornelia C. Metges ${ }^{2}\left(\mathbb{D}\right.$ and Steffen Maak ${ }^{1(1)}$ \\ 1 Institute of Muscle Biology and Growth, Leibniz Institute for Farm Animal Biology (FBN), \\ 18196 Dummerstorf, Germany; zhao@fbn-dummerstorf.de (Y.Z.); maak@fbn-dummerstorf.de (S.M.) \\ 2 Institute of Nutritional Physiology “Oskar Kellner”, Leibniz Institute for Farm Animal Biology (FBN), \\ 18196 Dummerstorf, Germany; sciascia@fbn-dummerstorf.de (Q.L.S.); li@fbn-dummerstorf.de (Z.L.); \\ goers@fbn-dummerstorf.de (S.G.); schregel@fbn-dummerstorf.de (J.S.); \\ metges@fbn-dummerstorf.de (C.C.M.) \\ * Correspondence: elke.albrecht@fbn-dummerstorf.de; Tel.: +49-381-68858
}

Received: 29 September 2020; Accepted: 24 October 2020; Published: 28 October 2020

Simple Summary: The lower survival rate and diminished overall growth and performance of low birth weight piglets is an economic and ethical issue in pig production. Adapted nutrition can help to compensate growth retardation, in particular, if nutrients in milk are not sufficient for newborn piglets. This study investigated the effects of oral glutamine supplementation during the first days after birth on skeletal muscle morphology of piglets with low or normal birth weight. The results indicated that glutamine supplementation changed the intramuscular amino acid concentration in the short term and influenced the muscle fiber size and abundance of myosin heavy chain isoforms within longissimus and semitendinosus muscle in neonatal piglets. Furthermore, differences in muscle fiber size and intramuscular lipid deposition were observed between low and normal birth weight piglets that disappeared in older piglets, independent of supplementation. The results indicated a delayed muscle development in low birth weight piglets. This delay can partly be compensated during growth by adequate nutrition.

Abstract: Adapted nutrition can improve the growth of low birth weight (LBW) piglets. Since maternal milk is thought to provide insufficient glutamine (Gln) for LBW piglets, the current study investigated the influence of Gln supplementation during the early suckling period on development and lipid deposition in skeletal muscle. The weight differences between LBW and normal birth weight (NBW) littermates persisted from birth to slaughter $(p<0.001)$. However, intramuscular Gln and Ala concentrations were altered in piglets according to the supplementation $(p<0.01)$. There were larger muscle fibers $(p=0.048)$ in Gln-supplemented piglets. Capillarization or nuclei number per muscle fiber was not influenced by birth weight (BiW) or Gln supplementation. Abundance of myosin heavy chain $(\mathrm{MYH})$ isoforms was slightly altered by Gln supplementation. LBW piglets had more lipid droplets than NBW piglets at day 5 of life in both muscles $(p<0.01)$. The differences decreased with age. Adipocyte development increased with age, but was not influenced by BiW or supplementation. The results indicate that BiW differences were accompanied by differences in lipid deposition and muscle fiber structure, suggesting a delayed development in LBW piglets. Supplementation with Gln may support piglets to overcome those disadvantages.

Keywords: amino acids; glutamine supplementation; intramuscular lipid deposition; low birth weight; myosin heavy chain isoforms; piglets; skeletal muscle morphology 


\section{Introduction}

The increased demand for pork has led to selection for larger litter sizes in pig production which has resulted in increased variation of piglet birth weight (BiW) within a litter [1]. About $15-20 \%$ of piglets in one litter are affected by low birth weight (LBW) or intrauterine growth restriction (IUGR) caused by inadequate maternal nutrition and insufficient placental functions [2,3]. These animals exhibit lower survival rates, slower postnatal growth and faster deposition of body fat in comparison with normal or heavy birth weight piglets [4]. Furthermore, LBW piglets have lower insulin sensitivity [5] and changes in liver lipid and amino acid (AA) metabolism [6]. Thus, IUGR piglets or LBW piglets have lower muscle growth and increased fat deposition [7], resulting in higher carcass lipid content and diminished meat quality at slaughter [2]. However, adapted nutrition can be used to help LBW piglets partially compensate for the associated negative impacts on growth and developmental retardation. It has previously been reported that milk-fed piglets benefit from additional milk intake, while milk has traditionally been thought to provide adequate amounts of all amino acids to neonates [8]. However, studies suggest that dietary glutamine (Gln) provided by milk may be inadequate for protein synthesis of piglets [9] and additional Gln requirements were postulated, especially for LBW piglets [10].

Glutamine, as the most abundant free AA in the body, has its primary source in skeletal muscle and plays an important part in the body growth [11]. It is an energy substrate for dividing cells, supplying intracellular protein turnover with ATP [11]. Furthermore, Gln provides nitrogen and carbon skeletons [12] for endogenous arginine synthesis, stimulates mammalian target of rapamycin (mTOR) pathway [13], takes part in the synthesis of glutathione [14], acts as a precursor for the synthesis of purine and pyrimidine nucleotides [11], is involved in maintaining intestinal barrier integrity and function and promotes cell proliferation [15]. Overall, Gln is of great importance in muscle tissue growth and a lack of Gln is possibly detrimental to normal development during the early postnatal period of piglets [10]. Studies have shown that it is possible to increase the muscle fiber number in piglets during the first days of life by nutrition $[16,17]$. Thus, stimulation of muscle fiber growth could improve development of skeletal muscle tissue und overall growth of piglets.

The high fat intake via maternal milk diet before weaning supplies the necessary energy for the build-up of body fat that occurs during this period in piglets. Piglets have almost no body fat at the beginning of their life compared to other mammals, e.g., human and guinea pig neonates, who have approximately $10 \%$ body fat at birth $[18,19]$. Until weaning, fat deposition increases tremendously because of the enhanced ability of piglets to assimilate and transfer dietary fat into fat cells $[20,21]$. After weaning, lipogenic activity is increased. As the growth rate in neonatal piglets is higher compared to other stages of life [22], sufficient nutrient supply is the most important issue during this period. A better understanding and improvement of LBW piglet management, in particular nutritional strategy, are therefore required for both animal welfare and productivity. As BiW is highly related to porcine meat quality, this study investigated the early development of the Musculus longissimus (MLD) and Musculus semitendinosus (MST), which are both well-established and common models in meat quality determination [23].

We hypothesized that oral Gln supplementation during the early postnatal phase can improve muscle development and body composition, particularly in LBW piglets. The current study focused on both the effects of BiW on the development of muscle morphology, including muscle fibers, intramuscular lipids, adipocytes and capillarization during the early postnatal period and on their modulation with Gln supplementation.

\section{Materials and Methods}

\subsection{Animals and Sampling}

All experimental procedures followed the Directive 2010/63/EU (European Convention for the Protection of Vertebrate Animals used for Experimental and Other Scientific Purposes) and were approved by the responsible State Office for Agriculture, Food Safety and Fishing Mecklenburg-Western 
Pomerania, Germany (permission No. 7221.3-1-026/16). Four groups were generated, consisting of 144 male German Landrace piglets, $72 \mathrm{LBW}$ (BiW: 0.8 to $1.2 \mathrm{~kg}$ ) and 72 normal birth weight (NBW) littermates (BiW: 1.4 to $1.8 \mathrm{~kg}$ ). The LBW piglets were defined as piglets with weight below the lowest BiW quartile of the Leibniz Institute for Farm Animal Biology pig facility (FBN, Dummerstorf, Germany) [24], from where the piglets were sourced. Only six piglets were classified as IUGR according to Amdi et al. [25]. The piglets were supplemented with either $1 \mathrm{~g} \mathrm{Gln} / \mathrm{kg}$ body weight between day 1 and 12 after birth (the day of birth was considered as day 0 ), or an isonitrogenous amount of alanine (Ala, $1.22 \mathrm{~g} / \mathrm{kg}$ body weight). The four groups were named LBW-GLN, NBW-GLN, LBW-ALA and NBW-ALA. The Gln dose from the current study was chosen based on the study published by Haynes et al. [26], who showed this dosage has beneficial effects in piglets aged 7-14 days. The supplemented Gln and Ala were prepared from fresh powder (AppliChem, Darmstadt, Germany and Ajinomoto, Tokyo, Japan) mixed with $2 \mathrm{~mL}$ water and were syringe-fed to the piglets three times every day at 07:00, 12:00 and 17:00. Free access to water and feed was provided to the sows during gestation and the lactation period. Twelve piglets per group were slaughtered at postnatal days 5, 12 and 26 (dpn-days post natum), respectively. All piglets were fed a dosage of 3 to $20 \mathrm{~mL}$ milk (depending on age of piglets; $150 \mathrm{~g} / \mathrm{L}$ water dissolved at $45^{\circ} \mathrm{C}$, Neopigg Rescuemilk 2.0, Provimi, Rotterdam, The Netherlands) and 33\% of Gln and Ala of their daily intake, respectively, $2 \mathrm{~h}$ before slaughter.

Piglets slaughtered at $26 \mathrm{dpn}$ were nursed by their respective dams and supplemented with either Gln or Ala until 12 dpn. From 14 dpn, the piglets had access to creep feed. Muscle tissue of the MLD and MST was taken immediately after slaughter separately for histology and for protein isolation, and snap frozen in liquid nitrogen. The tissue samples were stored at $-80^{\circ} \mathrm{C}$ for further experiments. Whole muscle cross sections from both muscles were cut about $1 \mathrm{~cm}$ thick and stored at $-20{ }^{\circ} \mathrm{C}$ for determination of muscle cross sectional area.

\subsection{Measurement of Free Amino Acid Concentrations within M. Longissimus}

Muscle tissue of $30 \mathrm{mg}$ fresh weight from each piglet was thawed and homogenized on ice with $300 \mu \mathrm{L}$ lysis buffer according to the method described by Nebendahl et al. [27] with slight modifications, i.e., Tris was replaced by $10 \mathrm{mM}$ Hepes, Igepal by Tween 20 and no Phospho-Stop solution was used. Samples were sonicated using an ultrasonic tip (Sonotrode MS 0.5, Carl Roth, Stuttgart, Germany) with 25 pulses for $0.5 \mathrm{~s}$ each and $80 \%$ amplitude, then centrifuged with $3000 \times \mathrm{g}$ for $10 \mathrm{~min}$ at $4{ }^{\circ} \mathrm{C}$, and stored at $-20{ }^{\circ} \mathrm{C}$ until further analysis. The supernatant, tenfold diluted by ultra-pure water, was used for HPLC analysis as described in detail by Kuhla et al. [28], but with a HyperClone column (Phenomenex, Aschaffenburg, Germany). All free amino acid (AA) concentrations are presented as $\mu \mathrm{mol}$ per $1 \mathrm{~kg}$ fresh weight $(\mu \mathrm{mol} / \mathrm{kg})$.

\subsection{Histology and Histochemistry}

Serial sections of frozen MLD and MST were cut $10 \mu \mathrm{m}$ thick with a cryostat microtome (CM3050 S, Leica, Bensheim, Germany). Standard protocols were used to stain intramuscular lipids with Oil Red O (Chroma Gesellschaft, Münster, Germany), to stain capillaries with alkaline phosphatase/eosin and nuclei with hematoxylin and eosin $(\mathrm{H} / \mathrm{E}$, hematoxylin: Dako, Glostrup, Denmark; eosin: Chroma Gesellschaft, Münster, Germany). An Olympus BX43 microscope (Olympus, Hamburg, Germany) equipped with a UC30 color camera and Cell^D imaging software (OSIS, Münster, Germany) was used to analyze intramyocellular lipid droplets, intramuscular adipocyte size, muscle fiber size, capillary size and density, as well as nuclei number. Respective self-made macro programs within the Cell` $\mathrm{D}$ software (OSIS, Münster, Germany) were used as described by Zitnan [29] and Dahl [30]. For analysis of intramyocellular lipid droplets, at least five randomly selected regions were analyzed for each piglet, accounting for a total area of about $0.75 \mathrm{~mm}^{2}$. To determine rare intramuscular adipocytes, a larger total area of about $1 \mathrm{~cm}^{2}$ was analyzed for each piglet. Capillary density and nuclei number were measured in three randomly selected regions with a size of about $1 \mathrm{~mm}^{2}$ and $0.1 \mathrm{~mm}^{2}$, respectively. Total apparent muscle fiber number was calculated by multiplication of muscle fiber number per 
$\mathrm{mm}^{2}$ and mean cross sectional area of both sides of the muscle tissue slices. Pictures of muscle slices including a ruler were taken by a digital camera (Coolpix 8700, Nikon, Düsseldorf, Germany) and measured with the interactive measurement module of the Cell^D software.

\subsection{Immunohistochemistry}

Muscle sections of MLD were cut $12 \mu \mathrm{m}$ thick using a cryostat microtome (CM3050 S, Leica, Bensheim, Germany) and stained with antibodies against four myosin heavy chain (MYH) isoforms, namely MYH1, 2, 4 and 7. Antibodies against MYH isoforms (antigen homology to porcine proteins >94\%) were purchased from antibodies-online (Aachen, Germany; ABIN6570793, ABIN2916107, ABIN6263466, and ABIN3043105). In brief, muscle sections were fixed in 4\% paraformaldehyde (Carl Roth, Karlsruhe, Germany) for $15 \mathrm{~min}$ and then washed $2 \times 5$ min with phosphate-buffered saline (PBS) and permeabilized with 0.1\% Triton X100 (Sigma-Aldrich, Munich, Germany) in PBS for $10 \mathrm{~min}$. After a blocking step with 10\% normal goat serum (NGS) in PBST for $15 \mathrm{~min}$, slides were incubated with primary antibodies (MYH1, 2, 4 and 7 diluted at 1:100 in PBST with 2\% NGS) for 2 h. Then, the slides were rinsed briefly and washed $3 \times$ in PBST for 10 min each. Secondary antibodies (1:1000, Alexa Fluor 488 goat anti-rabbit IgG for MYH1, 2 and 4, Alexa Fluor 594 goat anti-mouse for MYH7, Life Technologies, Darmstadt, Germany) were incubated for $45 \mathrm{~min}$ in the dark. After that, the slides were rinsed briefly and washed in PBS for $3 \times 10 \mathrm{~min}$ and the nuclei were stained with Hoechst 33258 (Sigma-Aldrich, Munich, Germany) for $5 \mathrm{~min}$. Finally, the slides were washed and covered with ProLong Antifade (Thermo Fisher Scientific, Schwerte, Germany) and respective coverslips (Carl Roth, Karlsruhe, Germany). All incubations were done at room temperature (RT) in a humidity chamber. Negative controls, omitting the primary antibody, were generated to detect unspecific bindings of the secondary antibodies. No unspecific binding was detected. A Cell^F image analysis system (OSIS, Münster, Germany) equipped with a Nikon Microphot SA fluorescence microscope (Nikon, Düsseldorf, Germany) and a CC-12 color camera was used to localize myosin isoforms in muscle cross sections.

\subsection{Western Blotting}

Proteins were extracted from the MLD and MST with CelLytic MT lysis reagent (Sigma-Aldrich, Munich, Germany) and protease inhibitor as described in detail by Liu et al. [31]. Protein abundance of MYH isoforms was determined with a Jess Simple Western system (ProteinSimple, San Jose, CA, USA) according to the manufacturer's instructions. Briefly, a mixture of $2 \mu \mathrm{L}(1 \mu \mathrm{g})$ protein sample, $2 \mu \mathrm{L}$ of $0.1 \times$ sample buffer and $1 \mu \mathrm{l}$ of $5 \times$ Mastermix was incubated at $95^{\circ} \mathrm{C}$, briefly vortexed and centrifuged, and placed on ice before pipetting into the plate. Primary antibodies (MYH1, 4 at 1:40, MYH2 at 1:25, MYH7 at 1:100) and secondary antibodies (rabbit IR and mouse NIR at 1:20; Bio-Techne, Wiesbaden, Germany) were diluted. Plates were loaded with protein mixture, diluted antibodies, buffers and protein ladder, as well as normalization reagent as loading control (ProteinSimple, San Jose, CA, USA). Then the procedure was performed with standard incubation times and signals were recorded in the NIR, IR and PN channel for the targets and total protein, respectively. The results were analyzed with the Compass for SW software (ProteinSimple, San Jose, CA, USA). Protein abundance was calculated by dividing the band volume of the target protein by the volume of total protein and given as relative protein abundance.

\subsection{Statistical Analysis}

All data were analyzed with SAS statistical software (Version 9.4, SAS Inst., Cary, NC, USA) by the analysis of variance (ANOVA) model using the MIXED procedure with BiW (LBW, NBW), supplementation (ALA, GLN), age $(5,12,26)$ and respective interactions as fixed factors and sow as a random factor. Least square means (LSmeans) and standard errors (SE) were calculated for each fixed effect and pairwise differences were tested by the Tukey-Kramer test. The SLICE statement of the MIXED procedure was used for partitioned analyses of the LSmeans for the interaction between BiW and supplementation within ages. Differences were considered significant if Tukey-Kramer adjusted 
$p \leq 0.05$ and a trend if $0.1>p>0.05$. Pearson correlation coefficients were calculated using the CORR procedure of SAS.

\section{Results}

\subsection{Slaughter Weights}

Low birth weight piglets were on average about 25\% lighter at birth than their NBW littermates (Figure 1). The weight difference remained significant until slaughter $(p<0.001)$. There was no supplementation effect detected $(p=0.539)$. Birth weight and slaughter weight were highly correlated in the 5 dpn piglets $(r=0.898, p<0.001)$ and moderately correlated in the 12 and 26 dpn piglets $(\mathrm{r}=0.663$ and 0.669 , respectively, $p<0.001)$.

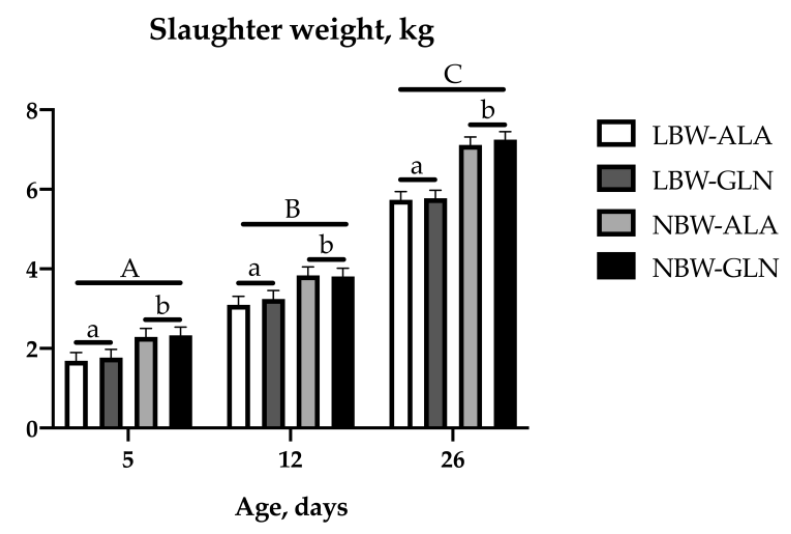

Figure 1. Slaughter weights of low birth weight (LBW) piglets and their normal birth weight (NBW) littermates supplemented with glutamine (GLN) or an isonitrogeneous amount of alanine (ALA) between 1 and 12 days post natum (dpn). Twelve piglets per group were each slaughtered at 5 , 12 , or $26 \mathrm{dpn}$; ${ }^{\mathrm{a}, \mathrm{b}}$ indicate significant differences between LBW and NBW piglets at the same age; A-C indicate significant differences among ages ( $p \leq 0.05$, Tukey-Kramer test).

\subsection{Concentrations of Free Amino Acids within M. Longissimus}

The concentrations of free AAs and their metabolites in MLD were measured to determine whether the supplemented AA reached the muscle tissue and could have modulated the cellular development during the early postnatal phase. Concentrations of intramuscular AAs and their metabolites are presented in Table 1. As intended, the Gln concentration was higher in MLD of Gln-supplemented piglets at 5 dpn $(p=0.001)$ compared to Ala-supplemented piglets. However, within the LBW animals, the Gln concentration was not significantly higher in supplemented piglets due to the high individual variation. No difference in Gln concentration was observed at $12 \mathrm{dpn}(p=0.166)$ or $26 \mathrm{dpn}$ $(p=0.373)$ between Gln- and Ala-supplemented animals. Similarly, the Ala concentration was higher in Ala-supplemented piglets at $5 \mathrm{dpn}(p<0.001)$ and at $12 \mathrm{dpn}(p<0.001)$, but this effect disappeared in piglets at $26 \mathrm{dpn}(p=0.730)$ compared to Gln supplementation. Concentrations of Gln and Ala metabolites were changed as well. Glutamic acid (Glu) tended to be lower at $12 \mathrm{dpn}(p=0.053)$, whereas citrulline (Cit) tended to be higher at $5 \mathrm{dpn}(p=0.069)$ in Gln-supplemented piglets. Moreover, BiW differences were found for aspartic acid (Asp, $p=0.022$ at $5 \mathrm{dpn}$ ), ornithine (Orn, $p=0.034$ at $12 \mathrm{dpn}$ ), proline (Pro, $p=0.003$ at $5 \mathrm{dpn}$ ) and glycine (Gly, $p=0.032$ at $5 \mathrm{dpn}$ and $p=0.047$ at $12 \mathrm{dpn}$ ) concentrations (Table 1). For indispensable AAs, concentration of leucine (Leu) tended to be higher at $5 \mathrm{dpn}(p=0.057)$ and was higher at $12 \mathrm{dpn}(p=0.001)$ in LBW piglets compared to NBW, while more isoleucine (Ile) and valine (Val) were measured in LBW piglets at $5(p=0.028, p=0.003)$ and $12 \mathrm{dpn}$ $(p=0.017, p<0.001)$. The lysine (Lys) concentration was higher in LBW compared to NBW piglets at 5 $(p=0.043)$ and $26 \mathrm{dpn}(p=0.004)$. Furthermore, more Val was measured in Gln- than Ala-supplemented piglets at $12 \mathrm{dpn}(p=0.033)$. On the other hand, the concentration of carnosine (Car) was higher 
( $p=0.007)$, while that of anserine (Anser) tended to be higher $(p=0.06)$ in Gln-supplemented compared to Ala-supplemented piglets at $12 \mathrm{dpn}$. The higher concentration of beta-alanine ( $\beta$-Ala) in LBW piglets at $5 \mathrm{dpn}(p=0.005)$ became a trend at $12 \mathrm{dpn}(p=0.053)$ and Anser concentration was lower in LBW piglets compared to NBW ones at $12(p=0.019)$ and $26 \mathrm{dpn}(p=0.001)$.

Table 1. Concentrations of intramuscular free amino acids and their metabolites ( $\mu \mathrm{mol} / \mathrm{kg}$ fresh weight) within M. longissimus of low (LBW) and normal birth weight (NBW) piglets supplemented with glutamine (GLN) or alanine (ALA) between 1 and $12 \mathrm{dpn}(n=12$ per group and age).

\begin{tabular}{|c|c|c|c|c|c|c|c|c|c|c|c|}
\hline \multirow{2}{*}{ Item } & \multirow{2}{*}{ Age } & \multirow{2}{*}{$\begin{array}{c}\text { LBW- } \\
\text { ALA }\end{array}$} & \multirow{2}{*}{$\begin{array}{c}\text { LBW- } \\
\text { GLN }\end{array}$} & \multirow{2}{*}{$\begin{array}{c}\text { NBW- } \\
\text { ALA }\end{array}$} & \multirow{2}{*}{$\begin{array}{c}\text { NBW- } \\
\text { GLN }\end{array}$} & \multirow{2}{*}{ SE } & \multirow{2}{*}{ Age } & \multirow{2}{*}{ SE } & \multicolumn{3}{|c|}{$p$-Value } \\
\hline & & & & & & & & & $\mathrm{BiW}$ & Sup & $\mathrm{BiW} \times \mathrm{Sup}$ \\
\hline \multirow{3}{*}{ Arg } & 5 & 126 & 144 & 134 & 176 & 47 & $145^{\mathrm{B}}$ & 23 & 0.670 & 0.520 & 0.882 \\
\hline & 12 & 534 & 468 & 414 & 526 & 47 & $495^{\mathrm{A}}$ & 23 & 0.282 & 0.950 & 0.089 \\
\hline & 26 & 131 & 160 & 134 & 92 & 47 & $129^{\mathrm{B}}$ & 23 & 0.493 & 0.888 & 0.782 \\
\hline \multirow{3}{*}{ Leu } & 5 & 153 & 146 & 133 & 129 & 12 & 140 & 8 & 0.057 & 0.634 & 0.269 \\
\hline & 12 & $154^{\mathrm{a}}$ & $153^{a}$ & $114^{\mathrm{b}}$ & $129^{a, b}$ & 13 & 138 & 9 & 0.001 & 0.538 & 0.007 \\
\hline & 26 & 146 & 153 & 143 & 143 & 12 & 146 & 8 & 0.501 & 0.727 & 0.866 \\
\hline \multirow{3}{*}{ Ile } & 5 & 138 & 137 & 124 & 108 & 10 & $127^{\mathrm{A}}$ & 6 & 0.028 & 0.401 & 0.107 \\
\hline & 12 & 82 & 99 & 63 & 71 & 11 & $79^{B}$ & 6 & 0.017 & 0.228 & 0.061 \\
\hline & 26 & 113 & 99 & 96 & 97 & 10 & $101^{\mathrm{A}}$ & 6 & 0.298 & 0.505 & 0.548 \\
\hline \multirow{3}{*}{ Lys } & 5 & 241 & 291 & 180 & 210 & 51 & $231^{\mathrm{B}}$ & 39 & 0.043 & 0.343 & 0.163 \\
\hline & 12 & 299 & 264 & 254 & 251 & 53 & $267^{\text {B }}$ & 41 & 0.409 & 0.664 & 0.780 \\
\hline & 26 & $444^{\mathrm{a}, \mathrm{b}}$ & $530^{a}$ & $396^{\mathrm{a}, \mathrm{b}}$ & $373^{b}$ & 49 & $436^{\mathrm{A}}$ & 36 & 0.004 & 0.490 & 0.010 \\
\hline \multirow{3}{*}{ Val } & 5 & $221^{a}$ & $207^{\mathrm{a}, \mathrm{b}}$ & $182^{b}$ & $182^{a, b}$ & 14 & 198 & 10 & 0.003 & 0.574 & 0.020 \\
\hline & 12 & $205^{a}$ & $226^{\mathrm{a}}$ & $159^{b}$ & $192^{a, b}$ & 13 & 195 & 10 & $<.001$ & 0.033 & $<.001$ \\
\hline & 26 & 181 & 167 & 175 & 171 & 14 & 173 & 9 & 0.904 & 0.486 & 0.862 \\
\hline \multirow{3}{*}{ Gln } & 5 & $5198^{a, b}$ & $6177^{a}$ & $4665^{b}$ & $5945^{a}$ & 357 & $5496^{\mathrm{A}}$ & 222 & 0.211 & 0.001 & 0.007 \\
\hline & 12 & 3682 & 4321 & 4006 & 4336 & 365 & $4086^{\text {B }}$ & 229 & 0.578 & 0.166 & 0.477 \\
\hline & 26 & 3393 & 2727 & 3047 & 3085 & 348 & $3063^{C}$ & 210 & 0.985 & 0.373 & 0.545 \\
\hline \multirow{3}{*}{ Ala } & 5 & $9453^{a}$ & $7360^{b}$ & $8738^{a}$ & $6935^{b}$ & 357 & $8122^{A}$ & 209 & 0.078 & $<.001$ & $<.001$ \\
\hline & 12 & $6436^{a}$ & $5075^{b}$ & $6402^{a}$ & $4885^{b}$ & 362 & $5700^{B}$ & 213 & 0.728 & $<.001$ & 0.001 \\
\hline & 26 & 3427 & 3533 & 3360 & 3499 & 350 & $3455^{\mathrm{C}}$ & 199 & 0.876 & 0.730 & 0.986 \\
\hline \multirow{3}{*}{ Glu } & 5 & 3934 & 4175 & 3908 & 3500 & 225 & $3879^{\mathrm{A}}$ & 140 & 0.070 & 0.700 & 0.101 \\
\hline & 12 & 3678 & 3291 & 3523 & 3055 & 230 & $3387^{\mathrm{B}}$ & 145 & 0.310 & 0.053 & 0.187 \\
\hline & 26 & 2204 & 2227 & 2222 & 2047 & 219 & $2175^{C}$ & 133 & 0.675 & 0.732 & 0.904 \\
\hline \multirow{3}{*}{ Asp } & 5 & 702 & 663 & 619 & 588 & 384 & $643^{\mathrm{A}}$ & 23 & 0.022 & 0.347 & 0.101 \\
\hline & 12 & 511 & 518 & 497 & 417 & 391 & $486^{\mathrm{B}}$ & 24 & 0.094 & 0.335 & 0.149 \\
\hline & 26 & 316 & 365 & 327 & 349 & 75 & $339^{\mathrm{C}}$ & 22 & 0.938 & 0.349 & 0.789 \\
\hline \multirow{3}{*}{ Cit } & 5 & 252 & 288 & 239 & 267 & 20 & $261^{\mathrm{A}}$ & 14 & 0.246 & 0.069 & 0.194 \\
\hline & 12 & 293 & 323 & 296 & 292 & 21 & $301^{A}$ & 15 & 0.343 & 0.456 & 0.424 \\
\hline & 26 & 153 & 146 & 138 & 120 & 19 & $139^{\mathrm{B}}$ & 13 & 0.173 & 0.506 & 0.486 \\
\hline \multirow{3}{*}{ Orn } & 5 & 142 & 175 & 173 & 150 & 38 & $160^{B}$ & 24 & 0.931 & 0.891 & 0.855 \\
\hline & 12 & $322^{a, b}$ & $364^{a}$ & $316^{\mathrm{a}, \mathrm{b}}$ & $233^{b}$ & 39 & $309^{\mathrm{A}}$ & 25 & 0.034 & 0.574 & 0.036 \\
\hline & 26 & 154 & 190 & 134 & 99 & 37 & $144^{\mathrm{B}}$ & 23 & 0.085 & 0.987 & 0.244 \\
\hline \multirow{3}{*}{ Pro } & 5 & $4766^{a, b}$ & $5164^{a}$ & $4132^{a, b}$ & $4099^{b}$ & 395 & $4541^{\mathrm{A}}$ & 293 & 0.003 & 0.588 & 0.019 \\
\hline & 12 & 1840 & 2373 & 2165 & 1822 & 410 & $2162^{B}$ & 309 & 0.686 & 0.704 & 0.113 \\
\hline & 26 & 1815 & 1804 & 1567 & 1798 & 380 & $1746^{\mathrm{B}}$ & 273 & 0.645 & 0.761 & 0.918 \\
\hline \multirow{3}{*}{ Gly } & 5 & 7347 & 7002 & 8123 & 7486 & 365 & $7490^{B}$ & 244 & 0.032 & 0.146 & 0.074 \\
\hline & 12 & 9537 & 9979 & 10284 & 10399 & 376 & $10080^{\mathrm{A}}$ & 254 & 0.047 & 0.419 & 0.176 \\
\hline & 26 & 7713 & 8161 & 7722 & 7458 & 354 & $7764^{\text {B }}$ & 229 & 0.234 & 0.795 & 0.394 \\
\hline
\end{tabular}


Table 1. Cont.

\begin{tabular}{|c|c|c|c|c|c|c|c|c|c|c|c|}
\hline \multirow{2}{*}{ Item } & \multirow{2}{*}{ Age } & \multirow{2}{*}{$\begin{array}{l}\text { LBW- } \\
\text { ALA }\end{array}$} & \multirow{2}{*}{$\begin{array}{c}\text { LBW- } \\
\text { GLN }\end{array}$} & \multirow{2}{*}{$\begin{array}{c}\text { NBW- } \\
\text { ALA }\end{array}$} & \multirow{2}{*}{$\frac{\text { NBW- }}{\text { GLN }}$} & \multirow{2}{*}{ SE } & \multirow{2}{*}{ Age } & \multirow{2}{*}{ SE } & \multicolumn{3}{|c|}{$p$-Value } \\
\hline & & & & & & & & & BiW & Sup & $\mathrm{BiW} \times \mathrm{Sup}$ \\
\hline \multirow{2}{*}{$\beta$-Ala } & 5 & 3051 & 3232 & 2585 & 2601 & 258 & $2867^{\mathrm{A}}$ & 182 & 0.005 & 0.667 & 0.041 \\
\hline & 26 & 863 & 531 & 762 & 552 & 249 & $677^{C}$ & 170 & 0.835 & 0.267 & 0.709 \\
\hline Car & 5 & 2774 & 3348 & 3093 & 3551 & 455 & $3192^{C}$ & 268 & 0.519 & 0.245 & 0.615 \\
\hline \multirow{3}{*}{ Anser } & 5 & 48 & 55 & 53 & 62 & 10 & $54^{C}$ & 8 & 0.362 & 0.313 & 0.592 \\
\hline & 12 & $129^{b}$ & $141^{\mathrm{a}, \mathrm{b}}$ & $141^{\mathrm{a}, \mathrm{b}}$ & $159^{a}$ & 10 & $142^{\mathrm{B}}$ & 8 & 0.019 & 0.060 & 0.028 \\
\hline & 26 & $268^{b}$ & $282^{a, b}$ & $296^{\mathrm{a}}$ & $297^{a}$ & 9 & $286^{\mathrm{A}}$ & 7 & 0.001 & 0.348 & 0.005 \\
\hline
\end{tabular}

BiW: birth weight; Sup: supplement; Arg: arginine; Leu: leucine; Ile: isoleucine; Lys: lysine; Val: valine; Gln: glutamine; Ala: alanine; Glu: glutamic acid; Asp: aspartic acid; Cit: citrulline; Orn: ornithine; Pro: proline; Gly: glycine; $\beta$-Ala: beta-alanine; Car: carnosine; Anser: anserine. Values represent least square means (LSmeans) and standard errors (SE), the largest $\mathrm{SE}$ is shown; ${ }^{\mathrm{a}, \mathrm{b}}$ indicate significant differences among groups at the same age; A-C indicate significant differences among ages ( $p \leq 0.05$, Tukey-Kramer test).

\subsection{Muscle Morphology and Capillarization}

Morphology and composition of two different muscles were investigated to determine whether the observed changes in AA concentrations and presumably higher availability of important AAs stimulated their development and growth. The LSmeans and SE for the BiW $\times$ Sup $\times$ Age interaction, $\mathrm{BiW} \times$ Sup over ages and for Age over groups are shown in Tables 2 and 3 for the MLD and MST, respectively. Muscle cross sectional area (CSA) reflected the differences in slaughter weights between LBW and NBW piglets. Overall, the CSA of both muscles was influenced by Age and BiW group but not by supplementation. Muscle CSA of MLD and slaughter weight correlated increasingly from $\mathrm{r}=0.422(p=0.003)$ at $5 \mathrm{dpn}$ to $\mathrm{r}=0.702(p<0.001)$ at $12 \mathrm{dpn}$ and $\mathrm{r}=0.835(p<0.001)$ at $26 \mathrm{dpn}$. Similar values were recorded for the MST $(r=0.41, p=0.004 ; r=0.71, p<0.001 ; r=0.78, p<0.001$ for 5, 12 and $26 \mathrm{dpn}$, respectively).

Table 2. Morphological traits in M. longissimus of low (LBW) and normal birth weight (NBW) piglets supplemented with glutamine (GLN) or alanine (ALA) between 1 and $12 \mathrm{dpn}(n=12$ per group and age).

\begin{tabular}{|c|c|c|c|c|c|c|c|c|c|c|c|}
\hline \multirow{2}{*}{ Trait } & \multirow{2}{*}{ Age } & \multirow{2}{*}{$\begin{array}{l}\text { LBW- } \\
\text { ALA }\end{array}$} & \multirow{2}{*}{$\begin{array}{l}\text { LBW- } \\
\text { GLN }\end{array}$} & \multirow{2}{*}{$\begin{array}{l}\text { NBW- } \\
\text { ALA }\end{array}$} & \multirow{2}{*}{$\begin{array}{l}\text { NBW- } \\
\text { GLN }\end{array}$} & \multirow{2}{*}{ SE } & \multirow{2}{*}{ Age } & \multirow{2}{*}{$\mathrm{SE}$} & \multicolumn{3}{|c|}{$p$-Value } \\
\hline & & & & & & & & & $\mathrm{BiW}$ & Sup & $\mathrm{BiW} \times \mathrm{Sup}$ \\
\hline \multirow{3}{*}{$\begin{array}{l}\text { Muscle cross } \\
\text { sectional area } \\
\left(\mathrm{mm}^{2}\right)\end{array}$} & 5 & 238.5 & 250.7 & 300.3 & 327.1 & 33.1 & $279.1^{C}$ & 24.4 & 0.004 & 0.492 & 0.030 \\
\hline & 26 & $635.4^{b}$ & $668.2^{b}$ & $791.8^{a}$ & $784.6^{a}$ & 32.0 & $720.0^{\mathrm{A}}$ & 22.7 & $<0.001$ & 0.675 & $<0.001$ \\
\hline & Overall & $426.1^{b}$ & $458.3^{b}$ & $529.8^{a}$ & $540.6^{a}$ & 19.2 & & & & & \\
\hline \multirow{3}{*}{$\begin{array}{l}\text { Muscle fiber } \\
\text { size }\left(\mu \mathrm{m}^{2}\right)\end{array}$} & 5 & 257.1 & 280.0 & 271.0 & 312.4 & 32.5 & $280.1^{C}$ & 20.8 & 0.393 & 0.297 & 0.584 \\
\hline & 26 & $538.5^{b}$ & $619.6^{\mathrm{a}, \mathrm{b}}$ & $594.8^{\mathrm{a}, \mathrm{b}}$ & $671.8^{a}$ & 31.7 & $606.2^{\mathrm{A}}$ & 19.6 & 0.048 & 0.014 & 0.021 \\
\hline & Overall & $396.5^{b}$ & $430.3^{\mathrm{a}, \mathrm{b}}$ & $423.7^{\mathrm{a}, \mathrm{b}}$ & $462.0^{\mathrm{a}}$ & 18.8 & & & & & \\
\hline \multirow{4}{*}{$\begin{array}{l}\text { Apparent total } \\
\text { muscle fiber } \\
\text { number }\left(\times 10^{5}\right)\end{array}$} & 5 & 8.9 & 9.6 & 10.9 & 11.4 & 0.9 & $10.2^{\mathrm{B}}$ & 0.6 & 0.022 & 0.562 & 0.129 \\
\hline & 12 & 10.5 & 12.2 & 12.3 & 12.8 & 0.9 & $11.9^{\mathrm{A}, \mathrm{B}}$ & 0.6 & 0.129 & 0.231 & 0.230 \\
\hline & 26 & 11.7 & 10.8 & 14.0 & 11.9 & 0.9 & $12.1^{\mathrm{A}}$ & 0.5 & 0.042 & 0.100 & 0.063 \\
\hline & Overall & 10.4 & 10.8 & 12.4 & 12.0 & 0.5 & & & & & \\
\hline $\begin{array}{l}\text { Number of } \\
\text { nuclei per } \\
\text { muscle fiber }\end{array}$ & Overall & 0.62 & 0.65 & 0.68 & 0.67 & 0.03 & & & & & \\
\hline
\end{tabular}


Table 2. Cont.

\begin{tabular}{|c|c|c|c|c|c|c|c|c|c|c|c|}
\hline Trait & Age & $\begin{array}{l}\text { LBW- } \\
\text { ALA }\end{array}$ & $\begin{array}{l}\text { LBW- } \\
\text { GLN }\end{array}$ & $\begin{array}{l}\text { NBW- } \\
\text { ALA }\end{array}$ & $\begin{array}{l}\text { NBW- } \\
\text { GLN }\end{array}$ & SE & Age & SE & \multicolumn{3}{|c|}{$p$-Value } \\
\hline \multirow{3}{*}{$\begin{array}{c}\text { Capillary } \\
\text { density } \\
\text { (number per } \\
\mathrm{mm}^{2} \text { ) }\end{array}$} & 5 & 84.1 & 88.8 & 98.9 & 100.3 & 11.3 & $93.0^{\mathrm{B}}$ & 7.5 & 0.149 & 0.772 & 0.527 \\
\hline & 26 & 133.7 & 121.5 & 140.8 & 123.8 & 11.0 & $130.0^{\mathrm{A}}$ & 7.1 & 0.602 & 0.187 & 0.149 \\
\hline & Overall & 110.5 & 115.9 & 120.7 & 123.8 & 6.5 & & & & & \\
\hline \multirow{3}{*}{$\begin{array}{l}\text { Capillary size } \\
\qquad\left(\mu \mathrm{m}^{2}\right)\end{array}$} & 5 & 81.2 & 87.6 & 86.5 & 99.3 & 9.5 & $88.7^{\mathrm{B}}$ & 5.9 & 0.298 & 0.290 & 0.500 \\
\hline & 26 & 100.6 & 113.6 & 93.3 & 96.0 & 9.3 & $100.9^{\mathrm{B}}$ & 5.6 & 0.129 & 0.404 & 0.334 \\
\hline & Overall & 108.5 & 107.7 & 106.7 & 106.3 & 5.5 & & & & & \\
\hline
\end{tabular}

BiW: birth weight; Sup: supplementation. Values represent LSmeans and standard errors (SE), the largest SE is shown; ${ }^{a, b}$ indicate significant differences among groups at the same age; ${ }^{\mathrm{A}-\mathrm{C}}$ indicate significant differences among ages ( $p \leq 0.05$, Tukey-Kramer test).

Muscle fiber size (Tables 2 and 3) was greater in piglets at $26 \mathrm{dpn}$ compared to animals at 5 and $12 \mathrm{dpn}$ in both muscles ( $p<0.001$, over groups). LBW piglets tended to have smaller muscle fibers than NBW piglets within MLD $(p=0.062)$ and within the MST $(p=0.088)$. Moreover, Gln-supplemented piglets had larger muscle fibers within MLD $\left(446.1 \mu \mathrm{m}^{2}, p=0.048\right)$ than Ala-supplemented animals $\left(410.1 \mu \mathrm{m}^{2}\right)$. The apparent total muscle fiber number of MLD was higher in $26 \mathrm{dpn}$ compared to $5 \mathrm{dpn}$ piglets ( $p=0.047$, over groups). In the MST, the apparent total muscle fiber number was higher in $26 \mathrm{dpn}$ than in 5 and $12 \mathrm{dpn}$ piglets $(p<0.001)$. LBW piglets had fewer $(p<0.01)$ muscle fibers (MLD: $10.6 \times 10^{5}$; MST: $7.0 \times 10^{5}$ ) compared to NBW piglets (MLD: $12.2 \times 10^{5}$; MST: $8.0 \times 10^{5}$ ) in both muscles. An influence of supplementation on the apparent total muscle fiber number was not detected.

Table 3. Morphological traits in M. semitendinosus of low (LBW) and normal birth weight (NBW) piglets supplemented with glutamine (GLN) or alanine (ALA) between 1 and $12 \mathrm{dpn}(n=12$ per group and age).

\begin{tabular}{|c|c|c|c|c|c|c|c|c|c|c|c|}
\hline Trait & Age & $\begin{array}{l}\text { LBW- } \\
\text { ALA }\end{array}$ & $\begin{array}{l}\text { LBW- } \\
\text { GLN }\end{array}$ & $\begin{array}{l}\text { NBW- } \\
\text { ALA }\end{array}$ & $\begin{array}{l}\text { NBW- } \\
\text { GLN }\end{array}$ & SE & Age & $\mathrm{SE}$ & \multicolumn{3}{|c|}{$p$-Value } \\
\hline \multirow{3}{*}{$\begin{array}{l}\text { Muscle cross } \\
\text { sectional area } \\
\left(\mathrm{mm}^{2}\right)\end{array}$} & 5 & 302.7 & 275.5 & 307.1 & 279.3 & 54.7 & $291.1^{B}$ & 44.3 & 0.900 & 0.509 & 0.928 \\
\hline & 26 & 605.3 & 543.8 & 644.1 & 659.3 & 52.3 & $613.1^{A}$ & 40.9 & 0.022 & 0.613 & 0.076 \\
\hline & Overall & 434.3 & 406.3 & 446.9 & 466.3 & 31.7 & & & & & \\
\hline \multirow{3}{*}{$\begin{array}{l}\text { Muscle fiber } \\
\text { size }\left(\mu \mathrm{m}^{2}\right)\end{array}$} & 5 & 375.6 & 407.1 & 434.2 & 460.5 & 42.6 & $419.4^{C}$ & 25.2 & 0.142 & 0.486 & 0.447 \\
\hline & 26 & 828.6 & 865.7 & 900.3 & 867.8 & 41.7 & $865.6^{\mathrm{A}}$ & 24.1 & 0.332 & 0.957 & 0.617 \\
\hline & Overall & 582.0 & 616.3 & 632.6 & 640.9 & 24.6 & & & & & \\
\hline \multirow{4}{*}{$\begin{array}{l}\text { Apparent total } \\
\text { muscle fiber } \\
\text { number }\left(\times 10^{5}\right)\end{array}$} & 5 & 0.39 & 0.41 & 0.53 & 0.57 & 0.09 & $0.47^{\mathrm{B}}$ & 0.07 & 0.012 & 0.661 & 0.084 \\
\hline & 12 & 0.61 & 0.61 & 0.74 & 0.70 & 0.09 & $0.66^{\mathrm{B}}$ & 0.07 & 0.061 & 0.794 & 0.290 \\
\hline & 26 & 1.20 & 0.98 & 1.17 & 1.13 & 0.08 & $1.12^{\mathrm{A}}$ & 0.06 & 0.338 & 0.096 & 0.115 \\
\hline & Overall & $0.73^{a, b}$ & $0.67^{b}$ & $0.81^{\mathrm{a}}$ & $0.80^{\mathrm{a}}$ & 0.05 & & & & & \\
\hline $\begin{array}{l}\text { Number of } \\
\text { nuclei per } \\
\text { muscle fiber }\end{array}$ & Overall & 0.65 & 0.65 & 0.68 & 0.76 & 0.03 & & & & & \\
\hline \multirow{4}{*}{$\begin{array}{l}\text { Capillary } \\
\text { density } \\
(\text { number } \\
\text { per } \mathrm{mm}^{2} \text { ) }\end{array}$} & 5 & 82.2 & 75.5 & 82.9 & 77.6 & 8.3 & 79.6 & 4.1 & 0.869 & 0.468 & 0.905 \\
\hline & 12 & 80.7 & 77.3 & 76.7 & 76.2 & 8.3 & 77.7 & 4.1 & 0.762 & 0.814 & 0.981 \\
\hline & 26 & $73.2^{a, b}$ & $84.7^{\mathrm{a}, \mathrm{b}}$ & $98.9^{a}$ & $65.8^{\mathrm{b}}$ & 8.3 & 80.6 & 4.1 & 0.684 & 0.197 & 0.031 \\
\hline & Overall & 78.7 & 79.2 & 86.2 & 73.2 & 4.8 & & & & & \\
\hline \multirow{4}{*}{$\begin{array}{l}\text { Capillary size } \\
\left(\mu \mathrm{m}^{2}\right)\end{array}$} & 5 & 200.3 & 211.8 & 230.9 & 225.1 & 17.8 & $217.0^{\mathrm{A}}$ & 10.3 & 0.177 & 0.869 & 0.543 \\
\hline & 12 & 199.1 & 188.6 & 172.8 & 205.5 & 18.1 & $191.5^{\mathrm{A}}$ & 10.5 & 0.774 & 0.534 & 0.523 \\
\hline & 26 & 135.8 & 109.2 & 114.8 & 128.2 & 17.5 & $122.0^{\mathrm{B}}$ & 9.8 & 0.948 & 0.711 & 0.646 \\
\hline & Overall & 178.4 & 169.9 & 172.8 & 186.3 & 10.3 & & & & & \\
\hline
\end{tabular}

BiW: birth weight; Sup: supplementation. Values represent LSmeans and standard errors (SE), the largest SE is shown; ${ }^{a, b}$ indicate significant differences among groups at the same age; ${ }^{\mathrm{A}-\mathrm{C}}$ indicate significant differences among ages ( $p \leq 0.05$, Tukey-Kramer test). 
Muscle sections stained with $\mathrm{H} / \mathrm{E}$ were used to determine the number of nuclei per area unit and per muscle fiber (Figure $2 \mathrm{a}-\mathrm{c}$ ). The nuclei number per $\mathrm{mm}^{2}$ (Tables 2 and 3 ) decreased with age from 5 dpn to 26 dpn significantly $(p<0.001)$. The muscle fiber size became larger at the same time, thus significant differences in the number of nuclei per muscle fiber were not detected $(p=0.105)$. There was only a trend for fewer myonuclei per muscle fiber within MLD in LBW compared to NBW piglets, independent of supplementation, at $5(p=0.087)$ and $26 \mathrm{dpn}(p=0.075)$, and within the MST in LBW piglets at $5(p=0.08)$ and $12 \mathrm{dpn}(p=0.068)$. No influence of Gln supplementation was detected $(p=0.168)$.

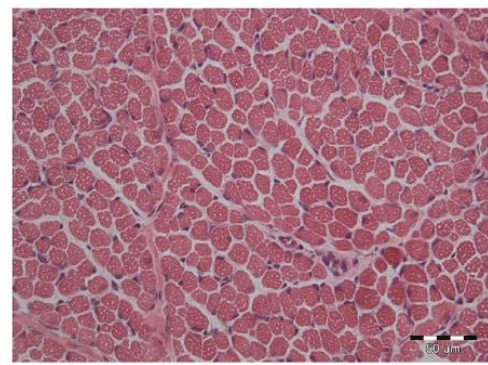

(a)

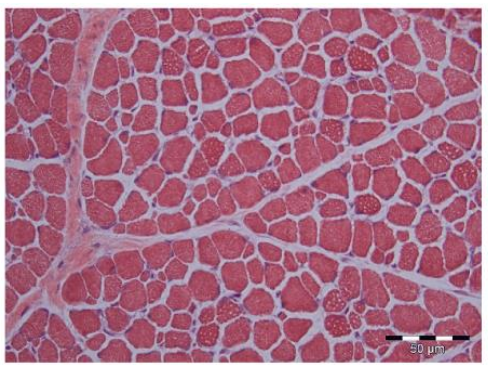

(b)

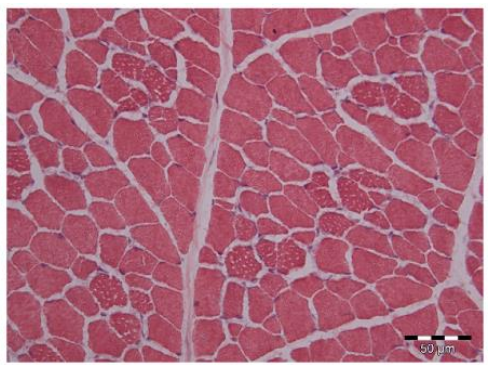

(c)

Figure 2. (a-c): Hematoxylin and eosin (H/E)-stained longissimus muscle cross sections of piglets at 5 , 12 and 26 dpn, respectively. Nuclei appear dark blue. Scale bar $=50 \mu \mathrm{m}$.

The capillarization was investigated to assess the potential nutrient and oxygen supply of the muscle by circulation and to detect possible effects of BiW and AA supplementation (Figure 3, Tables 2 and 3). The results indicated a trend for fewer capillaries ( $p=0.085$ ) in MLD of LBW piglets (113.2 per $\mathrm{mm}^{2}$ ) compared to NBW piglets (122.3 per $\mathrm{mm}^{2}$ ) over ages, independent of supplementation. Capillary density was lower in piglets at 5 dpn than in piglets at $12(p=0.004)$ and $26 \mathrm{dpn}(p=0.003)$. Capillary size in MLD was smaller $(p=0.04)$ at $12 \mathrm{dpn}$ in Gln-supplemented compared to Ala-supplemented piglets (Table 2). Capillary size was greater in piglets at $12 \mathrm{dpn}$ compared to $5 \mathrm{dpn}(p<0.001)$ and $26 \mathrm{dpn}(p=0.001)$, over ages, independent of supplementation. The capillary density in the MST was lower in NBW-GLN piglets compared to NBW-ALA piglets at 26 dpn $(p=0.028)$ and was not affected by age $(p=0.879)$. Capillary size was larger $(p<0.001)$ in piglets at 5 and 12 dpn compared to $26 \mathrm{dpn}$.

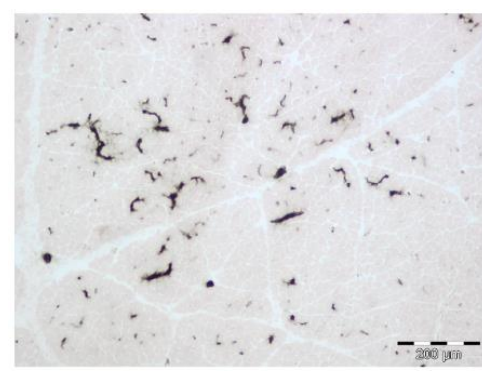

(a)

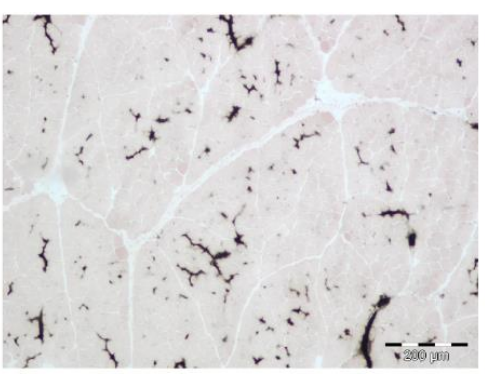

(b)

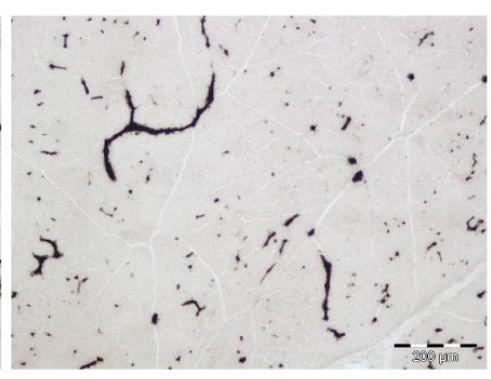

(c)

Figure 3. $(\mathbf{a}-\mathbf{c})$ : Alkaline phosphatase/eosin stained longissimus muscle cross sections of piglets at 5 , 12 and 26 dpn, respectively. Capillaries were stained black. Scale bar $=200 \mu \mathrm{m}$.

\subsection{Abundance of MYH Isoforms}

Myosin heavy chain isoforms were localized in muscle sections with immunohistochemistry, as shown in Figure 4. Fast MYH isoforms (MYH1 and 2) were predominant in MLD in 5 dpn and 26 dpn piglets (Figure 4a-d), while slow myosin was less abundant and usually observed in different muscle fibers (Figure 4e,f). Fibers expressing MYH2 and MYH7 at the same time were also detected 
(arrows in Figure 4g,h), suggesting transition from one fiber type to the other. The antibody against MYH4 did not show a specific staining pattern with immunohistochemistry, while it worked well in western blot.

5 dpn

MYH1

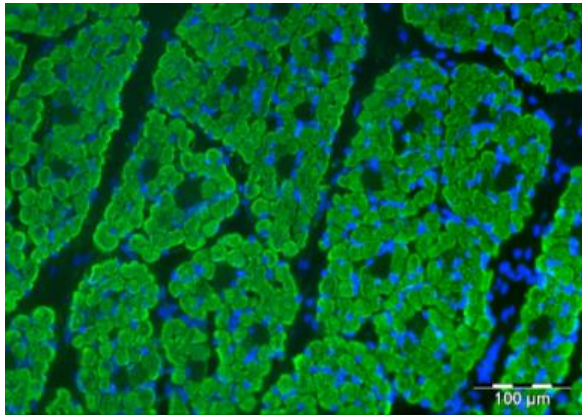

(a)

MYH2

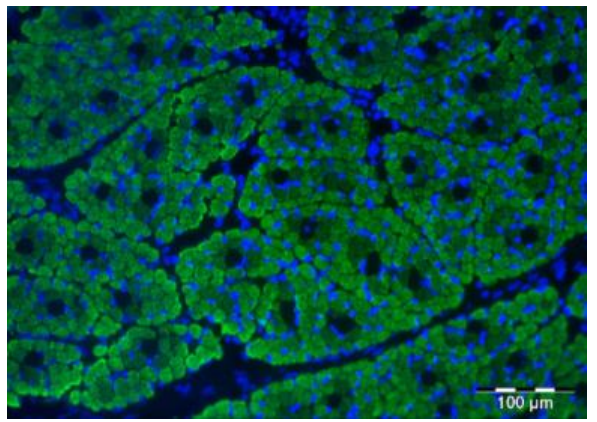

(c)

MYH7

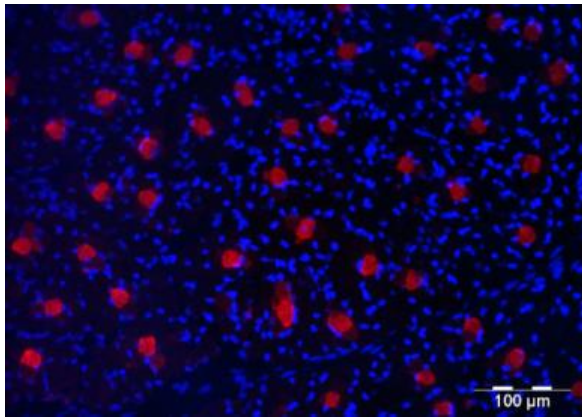

(e)

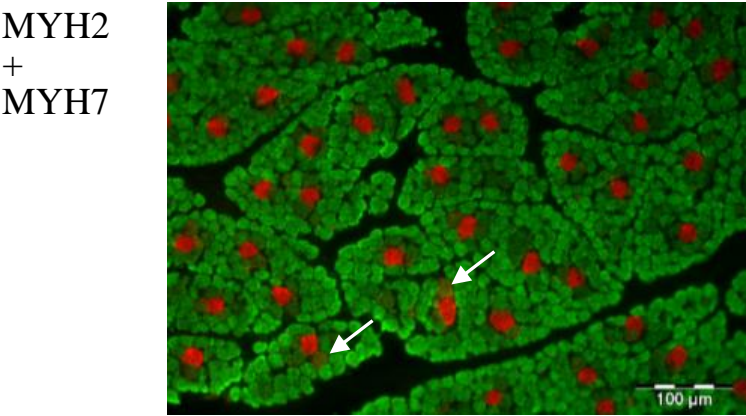

$(\mathrm{g})$
26 dpn

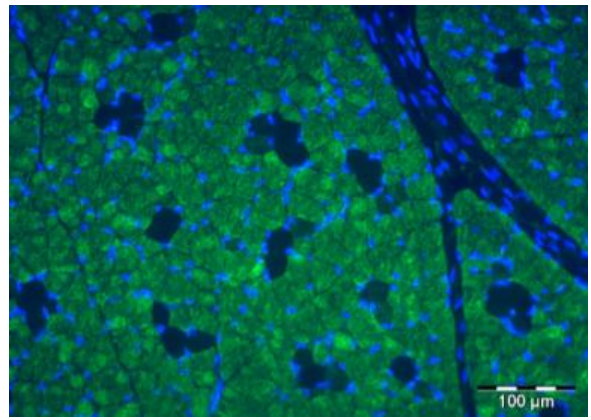

(b)

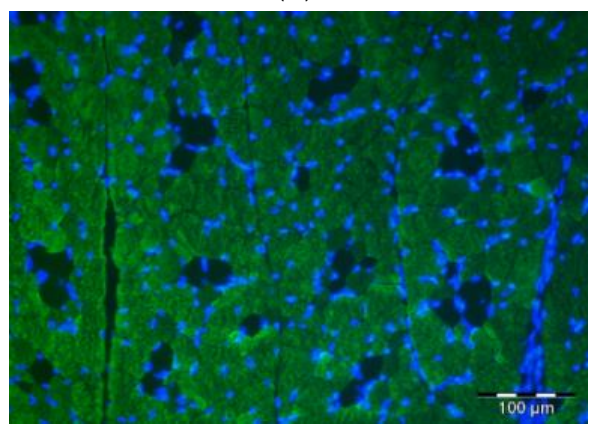

(d)

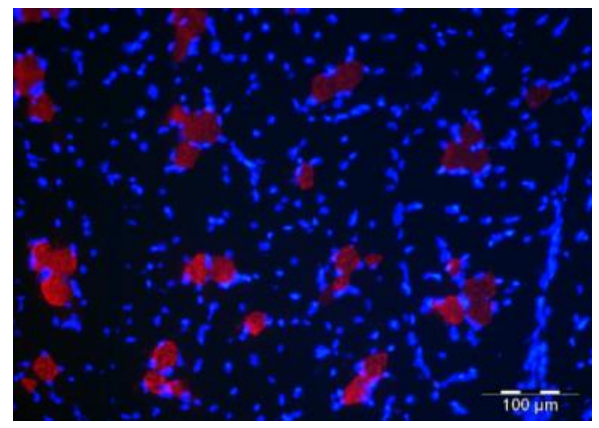

(f)

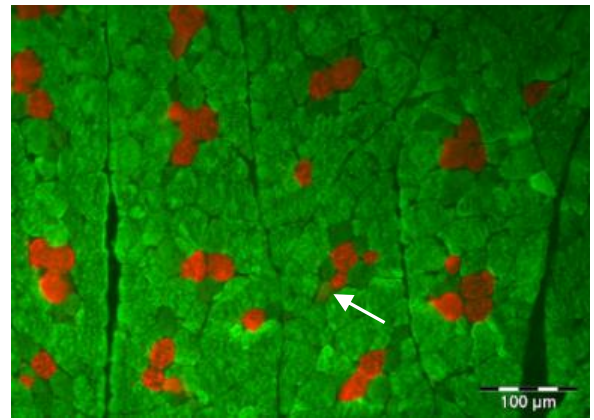

(h)

Figure 4. Localization of myosin heavy chain (MYH) isoforms within M. longissimus of piglets at 5 $(\mathbf{a}, \mathbf{c}, \mathbf{e}, \mathbf{g})$ and $26 \mathrm{dpn}(\mathbf{b}, \mathbf{d}, \mathbf{f}, \mathbf{h})$. MYH1 (a,b) and MYH2 (c,d,g,h) were stained green; MYH7 (e-h) was stained red. Nuclei were counterstained with Hoechst 33258 and appear in blue. Arrows in MYH2 and MYH7 double staining images $(\mathbf{g}, \mathbf{h})$ indicate transient fibers. Scale bar $=100 \mu \mathrm{m}$. 
Results of protein abundance of MYH isoforms are shown as LSmeans and SE for the $\mathrm{BiW} \times \operatorname{Sup} \times$ Age interaction, BiW $\times$ Sup over ages, and for Age over groups in Tables 4 and 5 for the MLD and MST, respectively. For MYH1 and MYH4 abundance in MLD, no influence of the single effects $\mathrm{BiW}(p=0.476, p=0.333)$, Gln supplementation $(p=0.769, p=0.895)$ or age $(p=0.552$, $p=0.392$ ) was detected. However, there was a trend for more MYH1 protein in LBW piglets at $12 \mathrm{dpn}$ $(p=0.074)$. The protein abundance of MYH2 was lower in MLD of piglets at $26 \mathrm{dpn}$ in comparison with piglets at $5 \mathrm{dpn}(p=0.026)$ and $12 \mathrm{dpn}(p=0.022)$. Compared to Ala-supplemented piglets, the protein abundance of MYH2 tended to be higher in Gln-supplemented piglets at $12 \mathrm{dpn}(p=0.055)$, but this trend was not observed in piglets at $5 \mathrm{dpn}(p=0.984)$ or $26 \mathrm{dpn}(p=0.882)$. In contrast, the MYH7 relative protein abundance in the MLD was not different between $26 \mathrm{dpn}$ and $5 \mathrm{dpn}$ $(p=0.228)$, and tended to be lower in Gln-supplemented piglets at $5 \mathrm{dpn}(p=0.065)$. Similar results were obtained for the MST (Table 5). No clear indications of BiW ( $p=0.709)$, supplementation $(p=0.949)$ or age ( $p=0.071)$ effects were found for MYH1 relative protein abundance in the MST. Furthermore, MYH2 protein abundance was lower in piglets at $26 \mathrm{dpn}$ compared to piglets at $12 \mathrm{dpn}(p=0.045)$ and $5 \mathrm{dpn}(p=0.062)$, but it was not influenced by BiW $(p=0.207)$ or Gln supplementation $(p=0.612)$. The MYH7 protein abundance was lower in NBW-GLN piglets compared to NBW-ALA piglets at $26 \mathrm{dpn}(p=0.006)$ and was lower in LBW-ALA than in NBW-ALA piglets $(p<0.001)$. MYH4 protein was not determined in the MST because of the unspecific staining pattern in MLD and the absence of differences among groups in western blot results of MLD.

Table 4. Relative normalized protein abundance of MYH isoforms in M. longissimus of low (LBW) and normal birth weight (NBW) piglets supplemented with glutamine (GLN) or alanine (ALA) between 1 and $12 \mathrm{dpn}$ ( $n=12$ per group and age).

\begin{tabular}{|c|c|c|c|c|c|c|c|c|c|c|c|}
\hline \multirow{2}{*}{ Item } & \multirow{2}{*}{ Age } & \multirow{2}{*}{$\begin{array}{l}\text { LBW- } \\
\text { ALA }\end{array}$} & \multirow{2}{*}{$\begin{array}{l}\text { LBW- } \\
\text { GLN }\end{array}$} & \multirow{2}{*}{$\begin{array}{l}\text { NBW- } \\
\text { ALA }\end{array}$} & \multirow{2}{*}{$\begin{array}{c}\text { NBW- } \\
\text { GLN }\end{array}$} & \multirow{2}{*}{ SE } & \multirow{2}{*}{ Age } & \multirow{2}{*}{$\mathrm{SE}$} & \multicolumn{3}{|c|}{$p$-Value } \\
\hline & & & & & & & & & $\mathrm{BiW}$ & Sup & $\mathrm{BiW} \times \mathrm{Sup}$ \\
\hline \multirow{4}{*}{ MYH1 } & 5 & 0.49 & 0.48 & 0.52 & 0.51 & 0.07 & 0.50 & 0.05 & 0.643 & 0.855 & 0.968 \\
\hline & 12 & 0.64 & 0.59 & 0.46 & 0.57 & 0.07 & 0.57 & 0.05 & 0.074 & 0.599 & 0.140 \\
\hline & 26 & 0.42 & 0.58 & 0.57 & 0.44 & 0.07 & 0.50 & 0.04 & 0.935 & 0.862 & 0.115 \\
\hline & Overall & 0.52 & 0.55 & 0.52 & 0.51 & 0.04 & & & & & \\
\hline \multirow{4}{*}{ MYH2 } & 5 & 0.51 & 0.51 & 0.53 & 0.54 & 0.06 & $0.52^{\mathrm{A}}$ & 0.04 & 0.619 & 0.984 & 0.969 \\
\hline & 12 & 0.49 & 0.52 & 0.47 & 0.64 & 0.06 & $0.53^{\mathrm{A}}$ & 0.04 & 0.210 & 0.055 & 0.051 \\
\hline & 26 & 0.35 & 0.36 & 0.42 & 0.39 & 0.06 & $0.38^{\mathrm{B}}$ & 0.04 & 0.240 & 0.882 & 0.662 \\
\hline & Overall & 0.45 & 0.46 & 0.48 & 0.52 & 0.03 & & & & & \\
\hline \multirow{4}{*}{ MYH4 } & 5 & 0.31 & 0.35 & 0.23 & 0.30 & 0.05 & 0.30 & 0.03 & 0.144 & 0.305 & 0.360 \\
\hline & 12 & 0.29 & 0.23 & 0.23 & 0.22 & 0.05 & 0.24 & 0.03 & 0.411 & 0.487 & 0.698 \\
\hline & 26 & 0.24 & 0.27 & 0.32 & 0.24 & 0.05 & 0.27 & 0.03 & 0.531 & 0.555 & 0.581 \\
\hline & Overall & 0.28 & 0.28 & 0.26 & 0.25 & 0.03 & & & & & \\
\hline \multirow{4}{*}{ MYH7 } & 5 & 20.31 & 12.73 & 13.95 & 13.02 & 2.58 & 15.00 & 1.83 & 0.117 & 0.065 & 0.035 \\
\hline & 12 & 9.44 & 14.26 & 13.96 & 11.60 & 2.62 & 12.32 & 1.92 & 0.628 & 0.579 & 0.262 \\
\hline & 26 & 8.57 & 10.18 & 10.27 & 13.53 & 2.51 & 10.64 & 1.71 & 0.211 & 0.310 & 0.421 \\
\hline & Overall & 12.77 & 12.39 & 12.73 & 12.72 & 1.49 & & & & & \\
\hline
\end{tabular}

MYH: myosin heavy chain; BiW: birth weight; Sup: supplementation. Values represent LSmeans and standard errors (SE), the largest SE is shown; ${ }^{A, B}$ indicate significant differences among ages ( $p \leq 0.05$, Tukey-Kramer test). 
Table 5. Relative normalized protein abundance of MYH isoforms in M. semitendinosus of low (LBW) and normal birth weight (NBW) piglets supplemented with glutamine (GLN) or alanine (ALA) between 1 and $12 \mathrm{dpn}$ ( $n=12$ for MYH7 and $n=6$ for MYH1 and 2 per group and age).

\begin{tabular}{|c|c|c|c|c|c|c|c|c|c|c|c|}
\hline \multirow{2}{*}{ Item } & \multirow{2}{*}{ Age } & \multirow{2}{*}{$\begin{array}{l}\text { LBW- } \\
\text { ALA }\end{array}$} & \multirow{2}{*}{$\begin{array}{l}\text { LBW- } \\
\text { GLN }\end{array}$} & \multirow{2}{*}{$\begin{array}{c}\text { NBW- } \\
\text { ALA }\end{array}$} & \multirow{2}{*}{$\begin{array}{l}\text { NBW- } \\
\text { GLN }\end{array}$} & \multirow{2}{*}{ SE } & \multirow{2}{*}{ Age } & \multirow{2}{*}{ SE } & \multicolumn{3}{|c|}{$p$-Value } \\
\hline & & & & & & & & & BiW & Sup & $\mathrm{BiW} \times \mathrm{Sup}$ \\
\hline \multirow{4}{*}{ MYH1 } & 5 & 0.39 & 0.38 & 0.33 & 0.40 & 0.05 & 0.38 & 0.04 & 0.529 & 0.511 & 0.533 \\
\hline & 12 & 0.34 & 0.33 & 0.32 & 0.33 & 0.05 & 0.33 & 0.04 & 0.800 & 0.996 & 0.953 \\
\hline & 26 & 0.42 & 0.42 & 0.51 & 0.44 & 0.05 & 0.45 & 0.03 & 0.128 & 0.517 & 0.250 \\
\hline & Overall & 0.38 & 0.38 & 0.39 & 0.39 & 0.03 & & & & & \\
\hline \multirow{4}{*}{ MYH2 } & 5 & 0.040 & 0.038 & 0.040 & 0.043 & 0.003 & $0.040^{\mathrm{A}, \mathrm{B}}$ & 0.002 & 0.177 & 0.782 & 0.319 \\
\hline & 12 & 0.042 & 0.038 & 0.038 & 0.041 & 0.002 & $0.040^{\mathrm{B}}$ & 0.002 & 0.898 & 0.770 & 0.193 \\
\hline & 26 & 0.046 & 0.045 & 0.045 & 0.049 & 0.002 & $0.046^{\mathrm{A}}$ & 0.002 & 0.340 & 0.367 & 0.289 \\
\hline & Overall & 0.042 & 0.040 & 0.041 & 0.044 & 0.001 & & & & & \\
\hline \multirow{4}{*}{ MYH7 } & 5 & 1.29 & 2.52 & 0.83 & 3.30 & 1.22 & 1.98 & 0.95 & 0.842 & 0.062 & 0.248 \\
\hline & 12 & 1.11 & 1.27 & 0.92 & 1.54 & 1.27 & 1.21 & 1.00 & 0.962 & 0.696 & 0.971 \\
\hline & 26 & $2.40^{\mathrm{b}}$ & $\begin{array}{c}3.69 \\
\mathrm{a}, \mathrm{b}\end{array}$ & $7.07^{\mathrm{a}}$ & $3.52^{b}$ & 1.17 & 4.17 & 0.88 & 0.006 & 0.294 & $<0.001$ \\
\hline & Overall & 1.60 & 2.50 & 2.94 & 2.79 & 0.70 & & & & & \\
\hline
\end{tabular}

MYH: myosin heavy chain; BiW: birth weight; Sup: supplementation. Values represent LSmeans and standard errors (SE), the largest $\mathrm{SE}$ is shown; ${ }^{\mathrm{a}, \mathrm{b}}$ indicate significant differences among groups at the same age; ${ }^{\mathrm{A}, \mathrm{B}}$ indicate significant differences among ages ( $p \leq 0.05$, Tukey-Kramer test).

\subsection{Intramuscular Lipid Deposition}

Intramuscular lipid deposition was quantified using Oil Red O-stained muscle slices. Two categories were separately analyzed, namely intramyocellular lipid droplets and lipids in developing adipocytes. Examples of stained muscle sections of MLD at 5, 12 and $26 \mathrm{dpn}$ are shown in Figure 5a-c. Intramyocellular lipid droplets decreased massively from $5 \mathrm{dpn}$ to $12 \mathrm{dpn}$ in both muscles $(p<0.001)$ and stayed unchanged thereafter until $26 \mathrm{dpn}(p=0.319$ and $p=0.51$, for MLD and MST, respectively, Figure 5d). Furthermore, LBW piglets had more lipid droplets within muscle fibers compared to NBW piglets at $5 \mathrm{dpn}$ in both muscles $(p<0.001$ and $p=0.032)$. For lipid droplets in developing adipocytes, greater area percentage was observed in piglets at $26 \mathrm{dpn}$ compared to younger piglets at 5 or $12 \mathrm{dpn}$ in both muscles ( $p \leq 0.001$, Figure 5e). Supplementation had no effect on lipid deposition ( $p=0.72$ and $p=0.91$, for MLD and MST, respectively). 


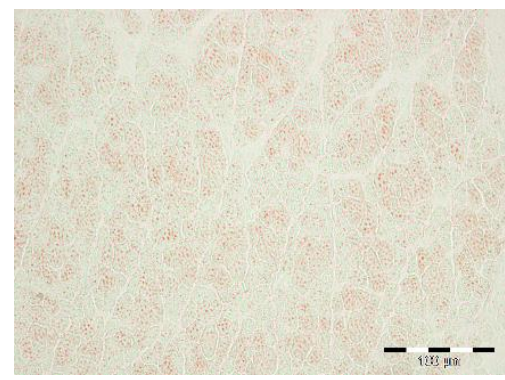

(a)

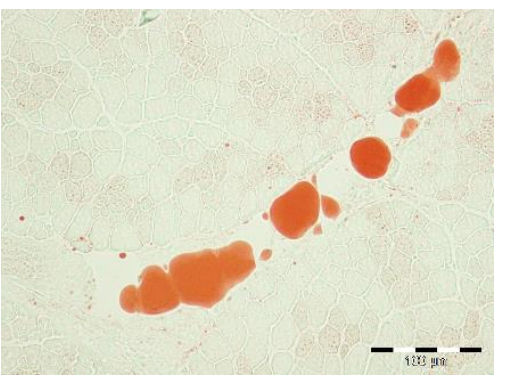

(b)

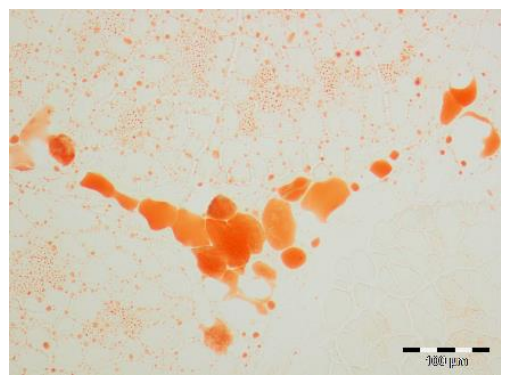

(c)

Intramyocelluar lipid droplets

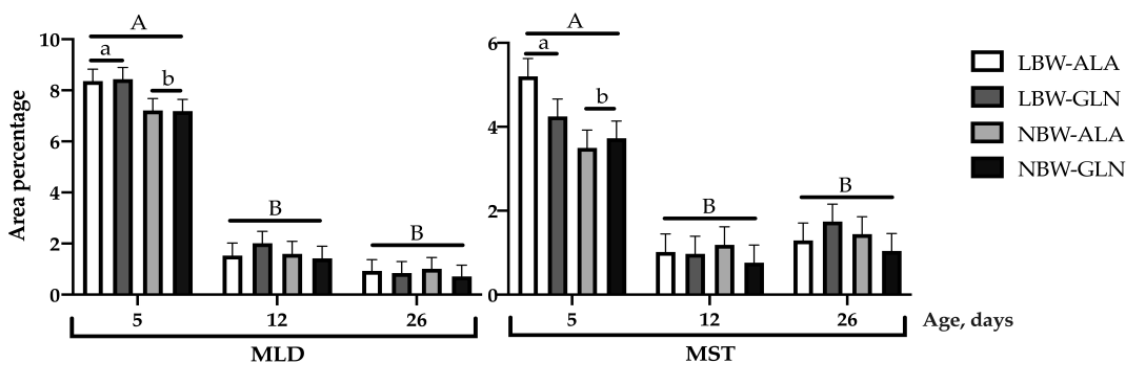

(d)

Lipids in adipocytes

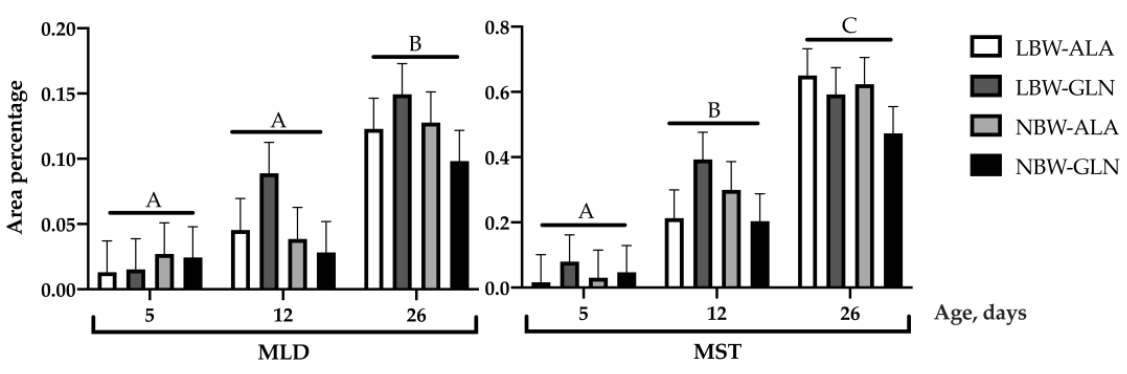

(e)

Figure 5. Intramuscular lipid deposition in M. longissimus (MLD) and M. semitendinosus (MST) of low (LBW) and normal birth weight piglets (NBW) supplemented with glutamine (GLN) or alanine (ALA) between 1 and $12 \mathrm{dpn}(n=12$ per group and age). (a-c): Oil Red O-stained longissimus muscle sections of piglets at 5,12 and $26 \mathrm{dpn}$, lipids are stained red. Scale bar $=100 \mu \mathrm{m}$. (d): Relative area of intramyocellular lipid droplets. (e): Relative area of intramuscular lipids in adipocytes. Values are LSmeans with SE in error bars; ${ }^{a}, \mathrm{~b}$ indicate significant differences among groups at the same age; A-C indicate significant differences among ages ( $p \leq 0.05$, Tukey-Kramer test).

\section{Discussion}

The dietary supplementation of AAs to pigs has received increasing interest in recent years $[9,15]$. For instance, arginine supplementation was shown to promote skeletal muscle growth, immunity and feed efficiency in young pigs [32-34] and supplemented branched chain AAs (Leu, Ile and Val) stimulated protein synthesis in skeletal muscle [35], while diets with Pro addition improved body growth of piglets [15]. Growth improvement has been achieved in some studies of dietary Gln supplementation in weaned piglets, but not in neonatal piglets, as reviewed by Wu et al. [10]. In weaned piglets with IUGR, Gln supplementation increased body weight gain and enhanced immune response [36]. The pigs in that study received a dosage of $1 \mathrm{~g} \mathrm{Gln} / \mathrm{kg}$ body weight twice a day, which is twofold higher than the dosage in our study with newborn piglets. Dosage and the developmental stage of the animals at which they receive the supplementation are obviously important factors for the 
achieved effects. In other species such as mice, Gln supplementation failed to promote weight gain [37]. Nevertheless, most of the studies focused on the intestine development under the influence of Gln supplementation, while skeletal muscle as the main site for Gln synthesis and storage in the body raised little attention [10]. Thus, the aim of the current study was to elucidate the effects of dietary Gln supplementation as well as BiW differences on skeletal muscle morphology of piglets during the early postnatal period. The sows in the experiment were all of the same parity (gilts), fed the same rations and raised under identical production conditions to reduce the number of influencing factors. Sow was also included in the statistical model as a random effect to account for groups of piglets being raised by the same sow. Additionally, milk composition from the sows was similar to previously published compositions (data not shown). Thus, the obtained results cannot be attributed to the sows' diet.

\subsection{Intramuscular Amino Acid Concentrations Were Altered by Oral Gln Supplementation}

Intramuscular Gln concentration was shown to be positively related to protein synthesis in rats $[38,39]$ and cultured chicken [40] skeletal muscle. Thus, our hypothesis was that a positive relationship exists between intracellular Gln concentration and skeletal muscle growth in young pigs. In perfused rat skeletal muscle, even though intramuscular Gln concentration was positively correlated with protein synthesis rate, its concentration had a limit of about $30 \mu \mathrm{mol} / \mathrm{g}$ on protein synthesis in the presence of insulin [38]. In our study, a maximum concentration of Gln of $5.2 \mu \mathrm{mol} / \mathrm{g}$ was observed. In previous studies, intracellular Gln concentration increased in jejunum and plasma in weanling piglets [41] and rats [42] by oral Gln supplementation. In addition, intramuscular Gln concentration was increased in 28-day-old weanling piglets supplemented with Gln for 7 days [43]. However, to our knowledge, few studies investigated consequences of the intramuscular Gln availability for skeletal muscle development, especially in pigs. In the present study, intramuscular Gln concentration was altered by Gln supplementation in piglets at $5 \mathrm{dpn}$, however, this effect was not visible in 12 and $26 \mathrm{dpn}$ piglets. We can only speculate that the ingested Gln was bound in the tissue and was not measurable as free AA. Thus, effects of supplementation were still possible. The cellular Ala and other free AA concentrations within MLD were influenced at a younger age of 5 and $12 \mathrm{dpn}$ as well. Therefore, supplementation had obviously short-term effects on the availability of essential AA in muscle tissue and might have affected muscle growth, particularly in early postnatal piglets, with some persistent or long-term effects. Furthermore, Leu, Ile and Val are components for Gln and Ala synthesis in skeletal muscle and other tissues, as reviewed by Wu et al. [15]. We observed higher Val concentrations in Gln-supplemented piglets at $12 \mathrm{dpn}$. Although the AAs were slightly influenced by supplementation, many AAs, e.g., Leu, Ile, Val, Asp, Pro, etc., were more abundant in LBW piglets at 5 or 12 dpn, indicating that these AAs were not sufficiently used in LBW for protein synthesis and muscle growth at a young age. The difference between LBW and NBW piglets disappeared at $26 \mathrm{dpn}$, suggesting that AAs were increasingly required for protein synthesis in LBW piglets. Furthermore, Asp, Val and Pro were reported to be more abundant in oxidative muscles [44,45]. This could be an evidence for more oxidative fibers in LBW piglets, which is discussed below.

\subsection{Muscle Fiber Size Was Influenced by Gln Supplementation}

In skeletal muscle, muscle fiber type, abundance of intramyocelluar lipid droplets, intramuscular adipocytes, nuclei and connective tissue, as well as capillary density, reflect growth performance of pigs and pork quality at the cellular level [46-48]. Generally, total muscle fiber number is fixed at birth in pigs, because primary and secondary fibers are formed during gestation [16], and the growth of muscle after birth is mainly determined by muscle fiber hypertrophy [49]. However, tertiary fibers with smaller size were discovered that develop after birth, even though they play a minor role in muscle development [16,50]. In the present study, we found greater apparent total muscle fiber numbers in both the MLD and MST at $26 \mathrm{dpn}$ compared to $5 \mathrm{dpn}$. The results indicate that there was a potential for increasing the size and number of muscle fibers in the early postnatal phase of piglets that can be supported by dietary supplementation. Consistent with Gondret's study [51], 
who reported that LBW piglets had fewer myofibers in the MLD compared to high birth weight piglets, we observed a trend for fewer muscle fibers in both muscles of LBW piglets in comparison with NBW littermates. Moreover, Gondret and colleagues found that LBW piglets had larger muscle fibers at $112 \mathrm{~kg}$ of body weight, but in young piglets in our study, muscle fibers tended to be smaller in LBW piglets. This suggests that LBW piglets catch up growth via increasing muscle hypertrophy and fat deposition [6,51]. Notably, the results of our study suggest that Gln supplementation promoted the growth of muscle fibers within the MLD and imply that dietary addition of Gln could stimulate piglets' skeletal muscle growth in early life. However, whether this greater muscle fiber size was caused by faster protein synthesis or extracellular fluid expansion from Gln supplementation [52] in the muscle tissue needs further investigations.

Capillary distribution can be an indicator of the nutrient and oxygen supply within skeletal muscle. It was hypothesized that capillarization is closely related to oxidative fibers [53,54]. Nonetheless, Maxwell et al. failed to find correlations between capillarization and oxidative capacity in several muscles of cats and other small mammals [55]. Bauer and colleagues $[55,56]$ found that the ratio of type I fibers to total fibers and the capillary density, as well as blood flow within muscle fibers, was greater in the flexor digitalis superficialis and gastrocnemius medialis of 1-day-old IUGR piglets compared to pigs with normal BiW. Additionally, clear correlations between type I fibers and capillarization were observed in flexor muscles [56]. However, in contrast to that study indicating that IUGR piglets have higher muscle blood supply [56,57], we still found a trend of smaller capillary density within the MLD in LBW piglets with no effects of supplementation or age, although our LBW piglets were not IUGR. Particular muscles may be differently affected by low BiW or IUGR. The trend for a lower capillarization of LBW piglets may explain the diminished supplementation effect on Gln concentration in the MLD. Therefore, BiW might be a potential factor influencing capillarization; however, we found no indication that Gln supplementation can modulate the capillary density development.

The nuclei within muscle fibers and associated satellite cells are the basis of muscle growth and regeneration [58] and can indicate the potential for muscle growth. In this study, myonuclei number per area unit declined with age in all groups, consistent with a previous study [59], which could be explained by hypertrophy of muscle fibers with increasing age. This negative relationship of nuclei number and muscle fiber size was also found in other studies [60]. LBW piglets tended to have fewer nuclei within both muscles at different ages, whereas the number of nuclei per muscle fiber remained almost constant until $26 \mathrm{dpn}$ in the present study. This, together with lower apparent total muscle fiber number in LBW piglets, could be an indication for the diminished muscle growth. Supplementation with Gln apparently did not alter the ratio of nuclei number per muscle fiber.

\subsection{Abundance of MYH Isoforms Was Slightly Altered by Gln Supplementation}

The four types of fibers in adult pig skeletal muscle are named type I, IIa, IIb and IIx, which are composed of different myosin heavy chain (MYH) isoforms [48] coded by MYH7, MYH2, MYH4 and MYH1 genes, respectively. Co-expression of different MYH isoforms forms hybrid muscle fibers [61], as was also shown in our study. In pig production, muscle fiber type composition is a factor influencing the meat quality [62]. More slow fibers promote tenderness, juiciness and flavor of pork [51,62]. However, compared to slow oxidative fibers, fast glycolytic fibers have a higher growth potential [49]. Therefore, it is contradictory to develop both body growth and meat quality in pigs at the same time. Our results indicated a trend for decreased MYH7 protein abundance upon Gln supplementation in the MLD of piglets at $5 \mathrm{dpn}$. Moreover, less MYH7 was measured in Gln-supplemented NBW piglets at 26 dpn within the MST, suggesting muscle fiber conversion from slow to fast type due to the supplementation. There was no strong evidence for this conversion from differences in MYH isoform abundance. However, we observed a higher intramuscular CAR concentration in Gln-supplemented piglets at $12 \mathrm{dpn}$, suggesting a higher number of fast glycolytic fibers, as CAR is usually enriched in those fibers [45]. Results of previous studies suggested that LBW piglets with IUGR had more slow muscle fibers within the flexor digitalis superficialis and gastrocnemius medialis [63]. Higher MYH7 
abundance could have pointed to the same direction in our study, but similar results were not observed in the MLD or MST of LBW piglets, even though higher oxidative fiber-related AA concentrations were observed in these animals. Thus, IUGR could be a factor influencing the muscle fiber types, in contrast to LBW without IUGR. Altogether, these results indicate that Gln supplementation and BiW had only minor effects on MYH protein abundance within skeletal muscle.

\subsection{Intramyocellular Lipid Droplets Were More Abundant in LBW Piglets}

Considering the lipogenesis in early postnatal piglets, lipid development competes with muscle growth [64], thus LBW piglets normally have a higher content of lipids and smaller muscle mass at slaughter age, as reviewed by Rehfeldt et al. [65]. Lipids in muscle are stored either within muscle fibers as intramyocellular lipid droplets or within adipocytes as intramuscular or marbling fat. Shortly after birth, piglets usually have no intramuscular fat [19]. All lipids are stored in intramyocellular lipid droplets, as observed in our study. A higher content of intramyocelluar lipid droplets was observed in LBW piglets compared to their NBW littermates at $5 \mathrm{dpn}$. Differences between LBW and NBW piglets disappeared with developing adipocytes, as seen from $12 \mathrm{dpn}$ onwards. However, there was no difference of intramuscular lipid content in adipocytes between LBW and NBW piglets in our study. The fat within skeletal muscle was not fully developed at these young ages, as also described in other reports [50]. An effect of Gln supplementation on lipid deposition within the MLD and MST was not observed. However, the obvious conversion of lipids within muscle tissue from intramyocellular droplets to adipocytes with increasing age and underlying factors, as well as potential modulators, might be an interesting topic for follow-up studies.

\section{Conclusions}

The current study confirmed that BiW influenced the skeletal muscle growth of neonatal piglets, as shown for muscle fiber size and apparent total muscle fiber number, as well as intramuscular lipid development. However, the Gln supplementation had some minor effects on modulating the muscle morphology during the investigated early postnatal period. Whether this is related to the used level of supplemented Gln cannot be determined from the present data. Nevertheless, potential Gln-associated changes in gene expression and cellular differentiation events warrant further investigation.

Author Contributions: Conceptualization, E.A., C.C.M.; methodology, Y.Z., E.A., S.G.; software, E.A.; validation, Y.Z., E.A., S.G., Q.L.S.; formal analysis, Y.Z., E.A.; investigation, Y.Z., E.A., Z.L., Q.L.S., S.G., J.S.; resources, C.C.M., E.A., S.M.; data curation, Y.Z., E.A.; writing-original draft preparation, Y.Z., E.A.; writing—review and editing, Y.Z., S.M, E.A., C.C.M., Z.L., Q.L.S., S.G., J.S.; supervision, S.M., E.A.; project administration, S.M., C.C.M.; funding acquisition, Y.Z., E.A., C.C.M. All authors have read and agreed to the published version of the manuscript.

Funding: Yaolu Zhao was funded by China Scholarship Council (CSC) grant. The project was partly funded by Deutsche Forschungsgemeinschaft (DFG), Bonn, Germany (grant number ME 1420/10-1). The publication of this article was funded by the Open Access Fund of the Leibniz Association and the Open Access Fund of the Leibniz Institute for Farm Animal Biology (FBN).

Acknowledgments: We thank E. Schwitulla, S. Foß, F. Feldt, K. Gürtler, K. Karpati, R. Pfuhl and the staff of the experimental slaughterhouse, as well as the EAS team for expert technical assistance and A. Tuchscherer for excellent statistical advice.

Conflicts of Interest: The authors declare that the research was conducted in the absence of any commercial or financial relationships that could be construed as a potential conflict of interest.

\section{References}

1. De Vos, M.; Che, L.; Huygelen, V.; Willemen, S.; Michiels, J.; Van Cruchten, S.; Van Ginneken, C. Nutritional interventions to prevent and rear low-birthweight piglets. J. Anim. Physiol. Anim. Nutr. 2014, 98, 609-619. [CrossRef] [PubMed]

2. Wu, G.; Bazer, F.W.; Wallace, J.M.; Spencer, T.E. Board-invited review: Intrauterine growth retardation: Implications for the animal sciences. J. Anim. Sci. 2006, 84, 2316-2337. [CrossRef] [PubMed] 
3. Alonso-Spilsbury, M.; Ramirez-Necoechea, R.; Gonzalez-Lozano, M.; Mota-Rojas, D.; Trujillo-Ortega, M.E. Piglet survival in early lactation: A review. J. Anim. Vet. Adv. 2007, 6, 76-86.

4. D'Inca, R.; Gras-Le Guen, C.; Che, L.Q.; Sangild, P.T.; Le Huerou-Luron, I. Intrauterine growth restriction delays feeding-induced gut adaptation in term newborn pigs. Neonatology 2011, 99, 208-216. [CrossRef] [PubMed]

5. Meas, T.; Deghmoun, S.; Alberti, C.; Carreira, E.; Armoogum, P.; Chevenne, D.; Levy-Marchal, C. Independent effects of weight gain and fetal programming on metabolic complications in adults born small for gestational age. Diabetologia 2010, 53, 907-913. [CrossRef] [PubMed]

6. Krueger, R.; Derno, M.; Goers, S.; Metzler-Zebeli, B.U.; Nuernberg, G.; Martens, K.; Pfuhl, R.; Nebendahl, C.; Zeyner, A.; Hammon, H.M.; et al. Higher body fatness in intrauterine growth retarded juvenile pigs is associated with lower fat and higher carbohydrate oxidation during ad libitum and restricted feeding. Eur. J. Nutr. 2014, 53, 583-597. [CrossRef] [PubMed]

7. Hegarty, P.V.; Allen, C.E. Effect of pre-natal runting on the post-natal development of skeletal muscles in swine and rats. J. Anim. Sci. 1978, 46, 1634-1640. [CrossRef] [PubMed]

8. Wu, G.; Bazer, F.W.; Cudd, T.A.; Meininger, C.J.; Spencer, T.E. Maternal nutrition and fetal development. J. Nutr. 2004, 134, 2169-2172. [CrossRef]

9. Wu, G. Amino acids: Metabolism, functions, and nutrition. Amino Acids 2009, 37, 1-17. [CrossRef]

10. Wu, G.; Bazer, F.W.; Johnson, G.A.; Knabe, D.A.; Burghardt, R.C.; Spencer, T.E.; Li, X.L.; Wang, J.J. Triennial Growth Symposium: Important roles for L-glutamine in swine nutrition and production. J. Anim. Sci. 2011, 89, 2017-2030. [CrossRef]

11. Curi, R.; Lagranha, C.J.; Doi, S.Q.; Sellitti, D.F.; Procopio, J.; Pithon-Curi, T.C.; Corless, M.; Newsholme, P. Molecular mechanisms of glutamine action. J. Cell Physiol. 2005, 204, 392-401. [CrossRef] [PubMed]

12. Wu, G.; Morris, S.M., Jr. Arginine metabolism: Nitric oxide and beyond. Biochem. J. 1998, 336 Pt 1, 1-17. [CrossRef]

13. Marc Rhoads, J.; Wu, G. Glutamine, arginine, and leucine signaling in the intestine. Amino Acids 2009, 37, 111-122. [CrossRef]

14. Wu, G.; Fang, Y.Z.; Yang, S.; Lupton, J.R.; Turner, N.D. Glutathione metabolism and its implications for health. J. Nutr. 2004, 134, 489-492. [CrossRef]

15. Wu, G.; Bazer, F.W.; Dai, Z.; Li, D.; Wang, J.; Wu, Z. Amino acid nutrition in animals: Protein synthesis and beyond. Annu. Rev. Anim. Biosci. 2014, 2, 387-417. [CrossRef]

16. Berard, J.; Kreuzer, M.; Bee, G. In large litters birth weight and gender is decisive for growth performance but less for carcass and pork quality traits. Meat Sci. 2010, 86, 845-851. [CrossRef]

17. Losel, D.; Kalbe, C.; Rehfeldt, C. L-Carnitine supplementation during suckling intensifies the early postnatal skeletal myofiber formation in piglets of low birth weight. J. Anim. Sci. 2009, 87, 2216-2226. [CrossRef] [PubMed]

18. Spray, C.M.; Widdowson, E.M. The effect of growth and development on the composition of mammals. Br. J. Nutr. 1950, 4, 332-353. [CrossRef] [PubMed]

19. Morise, A.; Louveau, I.; Le Huerou-Luron, I. Growth and development of adipose tissue and gut and related endocrine status during early growth in the pig: Impact of low birth weight. Animal 2008, 2, 73-83. [CrossRef]

20. Farnworth, E.R.; Kramer, J.K.G. Fat-Metabolism in Growing Swine-A Review. Can. J. Anim. Sci. 1987, 67, 301-318. [CrossRef]

21. Dodson, M.V.; Jiang, Z.; Du, M.; Hausman, G.J. Adipogenesis: It is not just lipid that comprises adipose tissue. J. Genom. 2013, 1, 1-4. [CrossRef] [PubMed]

22. Davis, T.A.; Fiorotto, M.L. Regulation of muscle growth in neonates. Curr. Opin. Clin. Nutr. 2009, 12, 78-85. [CrossRef] [PubMed]

23. Pardo, C.E.; Berard, J.; Kreuzer, M.; Bee, G. Intrauterine crowding impairs formation and growth of secondary myofibers in pigs. Animal 2013, 7, 430-438. [CrossRef]

24. Rehfeldt, C.; Lang, I.S.; Gors, S.; Hennig, U.; Kalbe, C.; Stabenow, B.; Brussow, K.P.; Pfuhl, R.; Bellmann, O.; Nurnberg, G.; et al. Limited and excess dietary protein during gestation affects growth and compositional traits in gilts and impairs offspring fetal growth. J. Anim. Sci. 2011, 89, 329-341. [CrossRef] 
25. Amdi, C.; Krogh, U.; Flummer, C.; Oksbjerg, N.; Hansen, C.F.; Theil, P.K. Intrauterine growth restricted piglets defined by their head shape ingest insufficient amounts of colostrum. J. Anim. Sci. 2013, 91, 5605-5613. [CrossRef] [PubMed]

26. Haynes, T.E.; Li, P.; Li, X.; Shimotori, K.; Sato, H.; Flynn, N.E.; Wang, J.; Knabe, D.A.; Wu, G. L-Glutamine or L-alanyl-L-glutamine prevents oxidant- or endotoxin-induced death of neonatal enterocytes. Amino Acids 2009, 37, 131-142. [CrossRef] [PubMed]

27. Nebendahl, C.; Kruger, R.; Gors, S.; Albrecht, E.; Martens, K.; Hennig, S.; Storm, N.; Hoppner, W.; Pfuhl, R.; Metzler-Zebeli, B.U.; et al. Effects on transcriptional regulation and lipid droplet characteristics in the liver of female juvenile pigs after early postnatal feed restriction and refeeding are dependent on birth weight. PLOS ONE 2013, 8, e76705. [CrossRef]

28. Kuhla, B.; Kucia, M.; Görs, S.; Albrecht, D.; Langhammer, M.; Kuhla, S.; Metges, C.C. Effect of a high-protein diet on food intake and liver metabolism during pregnancy, lactation and after weaning in mice. Proteomics 2010, 10, 2573-2588. [CrossRef]

29. Zitnan, R.; Albrecht, E.; Kalbe, C.; Miersch, C.; Revajova, V.; Levkut, M.; Rontgen, M. Muscle characteristics in chicks challenged with Salmonella Enteritidis and the effect of preventive application of the probiotic Enterococcus faecium. Poult. Sci. 2019, 98, 2014-2025. [CrossRef]

30. Dahl, N.; Albrecht, E.; Dannenberger, D.; Uken, K.L.; Hammon, H.M.; Maak, S. Consequences of maternal essential fatty acid and conjugated linoleic acid supplementation on the development of calf muscle and adipose tissue. Animals 2020, 10, 1598. [CrossRef]

31. Liu, Y.; Albrecht, E.; Schering, L.; Kuehn, C.; Yang, R.; Zhao, Z.; Maak, S. Agouti Signaling Protein and Its Receptors as Potential Molecular Markers for Intramuscular and Body Fat Deposition in Cattle. Front Physiol. 2018, 9, 172. [CrossRef] [PubMed]

32. Yao, K.; Yin, Y.L.; Chu, W.; Liu, Z.; Deng, D.; Li, T.; Huang, R.; Zhang, J.; Tan, B.; Wang, W.; et al. Dietary arginine supplementation increases mTOR signaling activity in skeletal muscle of neonatal pigs. J. Nutr. 2008, 138, 867-872. [CrossRef]

33. Tan, B.; Li, X.G.; Kong, X.; Huang, R.; Ruan, Z.; Yao, K.; Deng, Z.; Xie, M.; Shinzato, I.; Yin, Y.; et al. Dietary L-arginine supplementation enhances the immune status in early-weaned piglets. Amino Acids 2009, 37, 323-331. [CrossRef] [PubMed]

34. Kim, S.W.; McPherson, R.L.; Wu, G.Y. Dietary arginine supplementation enhances the growth of milk-fed young pigs. FASEB J. 2004, 18, A377-A378. [CrossRef] [PubMed]

35. Li, F.; Yin, Y.; Tan, B.; Kong, X.; Wu, G. Leucine nutrition in animals and humans: mTOR signaling and beyond. Amino Acids 2011, 41, 1185-1193. [CrossRef] [PubMed]

36. Zhong, X.; Li, W.; Huang, X.; Wang, Y.; Zhang, L.; Zhou, Y.; Hussain, A.; Wang, T. Effects of glutamine supplementation on the immune status in weaning piglets with intrauterine growth retardation. Arch. Anim. Nutr. 2012, 66, 347-356. [CrossRef]

37. Chen, S.; Xia, Y.; Zhu, G.; Yan, J.; Tan, C.; Deng, B.; Deng, J.; Yin, Y.; Ren, W. Glutamine supplementation improves intestinal cell proliferation and stem cell differentiation in weanling mice. Food Nutr. Res. 2018, 62. [CrossRef]

38. Maclennan, P.A.; Brown, R.A.; Rennie, M.J. A positive relationship between protein synthetic rate and intracellular glutamine concentration in perfused rat skeletal-muscle. FEBS Lett. 1987, 215, 187-191. [CrossRef]

39. Jepson, M.M.; Bates, P.C.; Broadbent, P.; Pell, J.M.; Millward, D.J. Relationship between glutamine concentration and protein-synthesis in rat skeletal-muscle. Am. J. Physiol. 1988, 255, E166-E172. [CrossRef]

40. Wu, G.Y.; Thompson, J.R. The effect of glutamine on protein turnover in chick skeletal muscle in vitro. Biochem. J. 1990, 265, 593-598. [CrossRef]

41. Wang, J.; Chen, L.; Li, P.; Li, X.; Zhou, H.; Wang, F.; Li, D.; Yin, Y.; Wu, G. Gene expression is altered in piglet small intestine by weaning and dietary glutamine supplementation. J. Nutr. 2008, 138, 1025-1032. [CrossRef]

42. Rogero, M.M.; Tirapegui, J.; Pedrosa, R.G.; Pires, I.S.D.; de Castro, I.A. Plasma and tissue glutamine response to acute and chronic supplementation with L-glutamine and L-alanyl-L-glutamine in rats. Nutr. Res. 2004, 24, 261-270. [CrossRef] 
43. Yoo, S.S.; Field, C.J.; McBurney, M.I. Glutamine supplementation maintains intramuscular glutamine concentrations and normalizes lymphocyte function in infected early weaned pigs. J. Nutr. 1997, 127, 2253-2259. [CrossRef] [PubMed]

44. Aristoy, M.C.; Toldrá, F. Concentration of free amino acids and dipeptides in porcine skeletal muscles with different oxidative patterns. Meat Sci. 1998, 50, 327-332. [CrossRef]

45. Cornet, M.; Bousset, J. Free amino acids and dipeptides in porcine muscles: Differences between 'red' and 'white' muscles. Meat Sci. 1999, 51, 215-219. [CrossRef]

46. Kriketos, A.D.; Pan, D.A.; Lillioja, S.; Cooney, G.J.; Baur, L.A.; Milner, M.R.; Sutton, J.R.; Jenkins, A.B.; Bogardus, C.; Storlien, L.H. Interrelationships between muscle morphology, insulin action, and adiposity. Am. J. Physiol. 1996, 270, R1332-R1339. [CrossRef] [PubMed]

47. Aniansson, A.; Hedberg, M.; Henning, G.B.; Grimby, G. Muscle morphology, enzymatic activity, and muscle strength in elderly men: A follow-up study. Muscle Nerve 1986, 9, 585-591. [CrossRef] [PubMed]

48. Listrat, A.; Lebret, B.; Louveau, I.; Astruc, T.; Bonnet, M.; Lefaucheur, L.; Picard, B.; Bugeon, J. How muscle structure and composition influence meat and flesh quality. Sci. World J. 2016, 2016, 3182746. [CrossRef]

49. Lefaucheur, L. A second look into fibre typing-Relation to meat quality. Meat Sci. 2010, 84, $257-270$. [CrossRef]

50. Stange, K.; Miersch, C.; Sponder, G.; Rontgen, M. Low birth weight influences the postnatal abundance and characteristics of satellite cell subpopulations in pigs. Sci. Rep. 2020, 10,1-14. [CrossRef]

51. Gondret, F.; Lefaucheur, L.; Juin, H.; Louveau, I.; Lebret, B. Low birth weight is associated with enlarged muscle fiber area and impaired meat tenderness of the longissimus muscle in pigs. J. Anim. Sci. 2006, 84, 93-103. [CrossRef] [PubMed]

52. House, J.D.; Pencharz, P.B.; Ball, R.O. Glutamine supplementation to total parenteral nutrition promotes extracellular fluid expansion in piglets. J. Nutr. 1994, 124, 396-405. [CrossRef] [PubMed]

53. Gray, S.D. Histochemical analysis of capillary and fiber-type distributions in skeletal muscles of spontaneously hypertensive rats. Microvasc. Res. 1988, 36, 228-238. [CrossRef]

54. Degens, H.; Turek, Z.; Hoofd, L.J.; Van't Hof, M.A.; Binkhorst, R.A. The relationship between capillarisation and fibre types during compensatory hypertrophy of the plantaris muscle in the rat. J. Anat. 1992, 180 (Pt 3), 455-463.

55. Maxwell, L.C.; White, T.P.; Faulkner, J.A. Oxidative capacity, blood-flow, and capillarity of skeletal-muscles. J. Appl. Physiol. 1980, 49, 627-633. [CrossRef]

56. Bauer, R.; Gedrange, T.; Bauer, K.; Walter, B. Intrauterine growth restriction induces increased capillary density and accelerated type I fiber maturation in newborn pig skeletal muscles. J. Perinat. Med. 2006, 34, 235-242. [CrossRef]

57. Bauer, R.; Wank, V.; Walter, B.; Blickhan, R.; Zwiener, U. Reduced muscle vascular resistance in intrauterine growth restricted newborn piglets. Exp. Toxicol. Pathol. 2000, 52, 271-276. [CrossRef]

58. Mesires, N.T.; Doumit, M.E. Satellite cell proliferation and differentiation during postnatal growth of porcine skeletal muscle. Am. J. Physiol. Cell Physiol. 2002, 282, C899-C906. [CrossRef]

59. Mulvaney, D.R.; Marple, D.N.; Merkel, R.A. Proliferation of skeletal-muscle satellite cells after castration and administration of testosterone propionate. Exp. Biol. Med. 1988, 188, 40-45. [CrossRef]

60. Cheek, D.B.; Holt, A.B.; Hill, D.E.; Talbert, J.L. Skeletal muscle cell mass and growth-Concept of deoxyribonucleic acid unit. Pediatr. Res. 1971, 5, 312-328. [CrossRef]

61. Pette, D.; Staron, R.S. Myosin isoforms, muscle fiber types, and transitions. Microsc. Res. Tech. 2000, 50, 500-509. [CrossRef]

62. Chang, K.C.; da Costa, N.; Blackley, R.; Southwood, O.; Evans, G.; Plastow, G.; Wood, J.D.; Richardson, R.I. Relationships of myosin heavy chain fibre types to meat quality traits in traditional and modern pigs. Meat Sci. 2003, 64, 93-103. [CrossRef]

63. Wank, V.; Bauer, R.; Walter, B.; Kluge, H.; Fischer, M.S.; Blickhan, R.; Zwiener, U. Accelerated contractile function and improved fatigue resistance of calf muscles in newborn piglets with IUGR. Am. J. Physiol. Regul. Integr. Comp. Physiol. 2000, 278, R304-R310. [CrossRef] [PubMed] 
64. Hocquette, J.F.; Gondret, F.; Baeza, E.; Medale, F.; Jurie, C.; Pethick, D.W. Intramuscular fat content in meat-producing animals: Development, genetic and nutritional control, and identification of putative markers. Animal 2010, 4, 303-319. [CrossRef] [PubMed]

65. Rehfeldt, C.; Kuhn, G. Consequences of birth weight for postnatal growth performance and carcass quality in pigs as related to myogenesis1. J. Anim. Sci. 2006, 84, E113-E123. [CrossRef] [PubMed]

Publisher's Note: MDPI stays neutral with regard to jurisdictional claims in published maps and institutional affiliations.

(C) 2020 by the authors. Licensee MDPI, Basel, Switzerland. This article is an open access article distributed under the terms and conditions of the Creative Commons Attribution (CC BY) license (http://creativecommons.org/licenses/by/4.0/). 